\title{
Arctic shipping: a systematic literature review of comparative studies
}

\begin{abstract}
Following the gradual decline of Arctic sea ice, shipping using Arctic routes increased from 2010. This led to an upsurge in the number of studies investigating the potential of Arctic maritime routes. A systematic literature review was conducted to assess the extant literature from 1980 to 2017 on comparative studies between Arctic and traditional routes. This review also aimed to provide an initial understanding on route choice decision-making factors and to contribute to the literature by providing suggestions for future research and methodological considerations. The competitiveness of Arctic routes is evaluated from both economic and environmental perspectives. Research themes and methodological characteristics are analysed in order to establish an evidence base in Arctic shipping literature. It is identified that analytical research methods and transport cost models are mainly employed. The results indicate that although Arctic routes can be more cost-effective and energy efficient compared to traditional ones, especially in the long-term, they can mainly serve as seasonal alternatives for bulk and specialised shipping in the short-term.
\end{abstract}

Keywords: systematic literature review; Arctic shipping; maritime routing; economic and environmental assessment; methodological characteristics; decision-making factors

\section{Introduction}

Arctic routes could reshape maritime transport geography with respect to global container shipping networks and tramp shipping. The implications for liner shipping could be a possible reconfiguration of existing networks or the launch of new ones depending on the origindestination (OD) pairs. For bulk and specialised shipping, this could mean the opening of new routes for transport of raw materials, refined products and refrigerated cargoes between ports in Northwest Europe, the Baltic and the Arctic to Northeast Asia. The gradual change in Arctic sea ice conditions could potentially open up opportunities for the more frequent use of polar routes. Increased accessibility could facilitate shorter transit times, lower fuel and overall costs, improve network connectivity and lower carbon dioxide $\left(\mathrm{CO}_{2}\right)$ and other greenhouse gas (GHG) emissions. According to recent studies projecting future accessibility to the Arctic, more routeing alternatives for both the Northern Sea Route (NSR) and the Northwest Passage (NWP) will become available by 2050 for non-ice class vessels. The Transpolar Sea Route (TSR) will also become accessible for Polar Class 6 (PC6) vessels by that time (Smith and Stephenson, 2013; Melia et al., 2016). The possibility of operating via the TSR increases by mid-century, even for non-ice class vessels (Melia et al., 2016).

According to Eguíluz et al. (2016), in 2014 shipping activity in Arctic waters accounted for 9.3\% of global shipping traffic (including domestic, destination and transit traffic). This included $5.9 \%$ of dry bulk and general cargo and $4.2 \%$ of liquid bulk cargo. A steep increase in transit traffic (i.e. voyages between the Atlantic and the Pacific) through the NSR was recorded between 2011 and 2014 followed by a sharp decline in 2015. The average duration of the sailing season was between 4 and 5 months (Zhang et al., 2016). To date domestic and destination shipping predominate with bulk and general cargo being the main drivers behind the emergence of this route (Zhang et al., 2016).

Arctic shipping is an emerging topic within maritime transport research, demonstrating an exponential increase in publications during the last ten years. Lasserre $(2014 ; 2015)$ identified 26 comparative studies between Arctic and traditional routes from 1991 to 2013. Meng et al. (2016) reviewed 25 studies regarding navigational and commercial perspectives. However, to 
date, there has not been any systematic literature review evaluating the economic feasibility of Arctic routes. Moreover, no account has been taken of studies reporting on the environmental assessment of these routes. Further, the aforementioned studies focus on research aspects and do not discuss the research methodological characteristics. The large number of discrepancies and differing assumptions regarding the parameters and results of the studies identified by Lasserre $(2014 ; 2015)$ stress the need to evaluate the literature in a systematic way in order to identify factors that affect the viability of Arctic routes and add complexity to the route choice decision-making process.

This study systematically reviews the extant literature regarding comparative studies between the Arctic and traditional routes from both economic (costs, profits) and environmental (emissions) perspectives between 1980 and 2017. The current state of Arctic shipping literature is evaluated in order to establish a new evidence base, and to suggest areas for future research, and various methodological approaches. This review also serves as the starting point for developing a conceptual framework of route choice decision-making factors which could be used in future research within the context of Arctic maritime routeing by including other sea and non-sea based routes.

The following research questions were formulated in order to address the objective of this study:

MAIN RQ: According to the extant literature, what is the cost effectiveness, and what is the likely impact on emissions, of using Arctic compared to traditional routes, between 1980 and 2017 ?

Two further sub-questions were developed for the purposes of this study:

RQ1: Which research methods and data analysis techniques are employed to address the research questions in comparative studies on Arctic shipping literature?

RQ2: What are the emerging issues that need to be addressed?

The remainder of this paper is organised as follows: First, the methodology used in the systematic review is explained. Subsequently, general statistics, methodological characteristics, and route choice decision-making factors are discussed and recommendations for future research made. Conclusions are drawn by reflecting on research gaps identified and methodological issues.

\section{Review methodology}

This study adopted the review design for systematic literature reviews in the field of management and business studies proposed by Tranfield et al. (2003). A systematic review of the literature is based on comprehensive and unbiased searches of relevant studies by explicitly formulating review questions and using specific search terms and inclusion criteria for that purpose. The findings are synthesised through various approaches in order to identify emerging themes, key results or any links to theory or concepts (Ibid, 2003). Traditional routes and oceanic canals dominate bulk shipping and determine connectivity in global liner networks. However, the emergence of new hubs (Notteboom, 2012), future canal development or expansion (Yip and Wong, 2016; Rodrigue and Ashar, 2016, Martinez et al., 2016) and the potential opening of polar routes (Tavasszy et al., 2011) could redefine the maritime transport geography landscape and increase maritime flows and network diversity (Ducruet, 2013). On the one hand, economic and environmental sustainability in shipping is achieved by employing vessels on traditional maritime routes and shipping canals. Different approaches to make 
shipping more cost-effective and greener include: slow steaming (e.g. Corbett et al., 2009; Notteboom and Vernimmen, 2009), scheduling optimisation (Lam, 2010), expansion of existing canals (De Marucci, 2012) or new ship sizes and designs (Knowles, 2006, Lindstad et al., 2013). On the other hand, Arctic routes could potentially become a viable alternative option to the classical shipping routes and canals so as to address both the economic and environmental sustainability in shipping, possibly by reducing the extent of the trade-offs involved between the latter two (Mansouri et al., 2015). All else being equal, the comparative advantage of Arctic routes stems from the fact that shorter geographical distances mean shorter transit times and operating costs, higher service frequency, potentially lower fuel consumption, which in turn means lower voyage costs, as well as lower $\mathrm{CO}_{2}$ and $\mathrm{GHG}$ emissions.

\subsection{Search strategy}

Arctic shipping literature contains studies spanning a broad spectrum of issues including economics, legal, geopolitics, geo-economics, climatic and technical and is informed by various disciplinary bases. The research scope of this review was narrowed according to the research questions so as to include only papers reporting original results on the economic (costs, profits) or environmental (emissions) assessment of Arctic routes compared to traditional ones. According to David and Han (2004), quality control is increased only by restricting the searches to journal papers and therefore excluding unpublished studies or book chapters.

Scopus was used for the initial scoping searches. Title, abstract and keywords of a sample of papers were searched covering all subject areas (fields of study) without specifying the period. The initial keywords used in Scopus were "arctic shipping "OR "northern sea route" OR "northwest passage", and resulted in 512 documents across a large range of disciplines. Abstracts of a sample of relevant papers were subsequently read and keywords were refined according to the aim and review questions of the study. Major shipping canals and maritime routes were used as keywords, as well as variations of terms that have similar meanings. The final set of keywords employed in the searches is presented in Table 1.

\begin{tabular}{lll}
\multicolumn{3}{l}{ Table 1. List of keywords used in the systematic review } \\
\hline arctic shipping & Nicaragua(n) Canal & Ship* canal(s) \\
Cape Horn & Northeast Passage & Ship* corridor(s) \\
Cape of Good Hope & Northwest Passage & Ship* lane(s) \\
Magellan Strait(s) & Northern Sea Route & Ship* passage(s) \\
Maritime canal(s) & Panama Canal & Ship* route(s) \\
Maritime corridor(s) & Sea canal(s) & Ship* strait(s) \\
Maritime lane(s) & Sea corridor(s) & Strait(s) of Magellan \\
Maritime passage(s) & Sea lane(s) & Suez Canal \\
Maritime route(s) & Sea passage(s) & transpolar passage \\
Maritime strait(s) & Sea route(s) & transpolar sea route \\
& Sea strait(s) & \\
\hline
\end{tabular}

*ship or shipping

Relevant publishers and databases were covered such as Elsevier, Emerald Insight, Taylor \& Francis, Cambridge Journals and Springer/Palgrave Macmillan. Two additional journals were identified: the Journal of Maritime Research (JMR) and the International Journal of Transport Economics (IJTE) that were not found in these databases. To ensure that the extant literature from 1980 to 2017 was covered, the searches were extended to include Thomson Reuters' database Web of Science, as well as the reference lists of the retrieved papers.

A total of 33 unique papers were retrieved and analysed based on their methodological and research considerations. Descriptive analysis is used to discuss the classification of the 
reviewed papers in publications per journal; total number of papers published per decade; total number of papers per country as well as the methodological considerations such as research methods and data analysis techniques reported in the data. Narrative synthesis is used for the analysis of the research considerations whilst the results are classified based on Stopford's cash flow model (Stopford, 2009).

\section{Results}

\subsection{General statistics}

Figure 1 shows the number of papers published from 1980-2017 included in the review. It is noticeable that a small number of papers assessed the potential of Arctic routes during the 1980s and most importantly from 1991 to 2000. Nevertheless, the lack of research interest during that period could be attributed to the underutilisation of Arctic routes and the lack of interest from the shipping industry in general. Of the 33 papers reviewed, two were published in the 1990s, seven between 2001 and 2010 and 24 between 2011 and 2017.

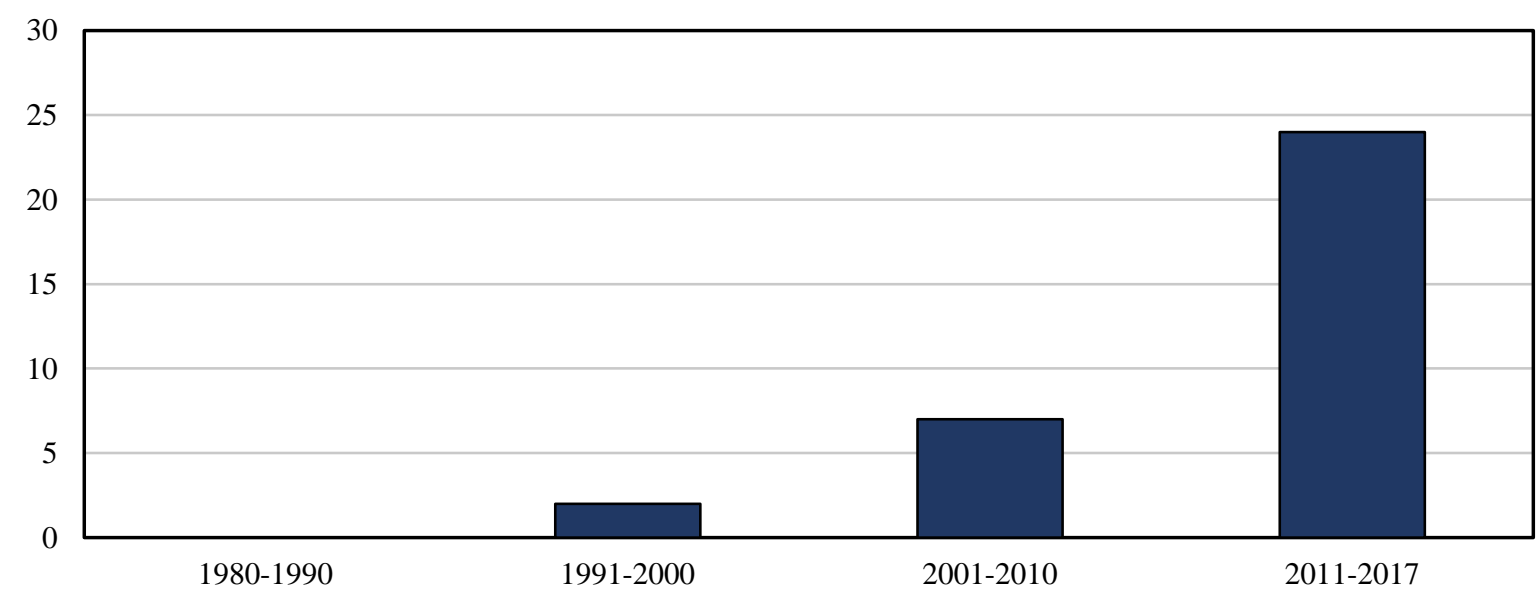

Fig. 1. Number of articles published between 1980 and 2017

This rising trend of publications appears to be consistent with the view that scholarly research followed the recent developments regarding the utilisation of Arctic routes. For instance, it was only in 2011 onwards that an increasing number of non-Russian flagged vessels started to use the Northern Sea Route (NSRIO, 2018). The 33 papers selected for this review were published in 22 journals. The Journal of Transport Geography and Transportation Research Part A: Policy and Practice have the most frequent publications followed by Maritime Policy \& Management and Maritime Economics \& Logistics, whereas the remaining journals each published one paper between 1992 and 2017 (Table 2).

Table 2. Number of articles published per academic journal

\begin{tabular}{lc}
\hline Country & No. of Articles \\
\hline Journal of Transport Geography & 5 \\
Transportation Research Part A: Policy and Practice & 5 \\
Maritime Policy \& Management & 3 \\
Maritime Economics \& Logistics & 2 \\
European Journal of Operational Research & 1 \\
International Journal or Production Economics & 1 \\
The Asian Journal of Shipping and Logistics & 1 \\
International Journal of e-Navigation and Maritime Economy & 1 \\
International Journal of Geographical Information Science & 1
\end{tabular}


$\begin{array}{lr}\text { Journal of Maritime Research } & 1 \\ \text { Transport Policy } & 1 \\ \text { Polar Record } & 1 \\ \text { Journal of Navigation } & 1 \\ \text { International Challenges } & 1 \\ \text { Journal of Ocean Technology } & 1 \\ \text { Applied Mechanics and Materials } & 1 \\ \text { Advanced Science Letters } & 1 \\ \text { Transportation Research Board } & 1 \\ \text { Ambio } & 1 \\ \text { Climatic Change } & 1 \\ \text { Izvestiya, Atmospheric and Oceanic Physics } & 1 \\ \text { Journal of Nuclear Science and Technology } & 1\end{array}$

Twelve countries have contributed to Arctic shipping research concerning the economic and environmental assessment of Arctic routes (Table 3). The selection of countries was based on the country of affiliation of the first author of each paper. Canada and China have the biggest contributions whereas Germany and Singapore have the lowest rate of contribution with one paper each.

Table 3. Contribution of publications based on country affiliation

\begin{tabular}{lc}
\hline Country & No. of Articles \\
\hline Canada & 5 \\
China & 5 \\
Norway & 4 \\
France & 3 \\
Taiwan & 3 \\
The Netherlands & 3 \\
Japan & 2 \\
Russia & 2 \\
USA & 2 \\
South Korea & 2 \\
Germany & 1 \\
Singapore & 1 \\
\hline
\end{tabular}

\subsection{Methodological considerations}

The categorisation extended to include the methodological characteristics of the reviewed papers, such as research methods and data analysis techniques. Arctic shipping is a topic within maritime transport research rather than a discipline per se. However, the differing assumptions reported in the extant literature regarding the cost assessments and determinant factors influencing route choice decision-making, as well as the growing trend of addressing the main research questions through different methodological perspectives, all stress the need to explore the methodological background of these studies apart from the research considerations.

\subsubsection{Research methods}

The categorisation scheme of research methods was adopted from Wacker (1998). In empirical research, data from the 'real world' are used in order to verify the relationships under investigation by using an inductive approach to theory, whereas in analytical research, logic, 
mathematics and/or statistics are primarily employed by using a deductive approach to theory to reach a conclusion (Wacker, 1998). Analytical mathematical methods (modelling or simulation) were reported in 27 papers, whereas empirical statistical and case studies were found in four and two papers respectively (Table 4).

Analytical modelling and simulation are used with the aim to develop mathematical relationships to explain the behaviour of real-world systems by investigating the performance of dependent variables or models under different conditions (Meredith et al., 1989). Papers that used transport cost models, optimisation or mathematical simulation techniques through case examples and Multi-Criteria Analysis belong to the category of analytical mathematical methods.

Empirical statistical research aims at verifying theoretical relationships by analysing large samples of data from real business processes (Wacker, 1998). Studies that employed regression analysis or structural modelling fall under this research methodology. Case studies focus on a specific phenomenon with the aim of revealing empirical relationships and usually serve for exploration in the early stages of research. They are also used to examine dependent variables under different scenarios or to provide counter-arguments to prior hypotheses or even to come up with new insights in debatable areas (Meredith et al., 1989). Papers classified under this research method have not made use of data analysis techniques.

\subsubsection{Data analysis techniques}

According to Sachan and Datta (2005), analytical techniques aid the researcher to deal with the summation of large amounts of data, identification of causal relationships and exploration of the effects on the outcome on alternative scenarios. Optimisation models were reported in four papers. Regression and Monte Carlo simulation accounted for three and two papers respectively, whereas logit model, Multi-Criteria Decision-Making (MCDM), GIS simulation and structural economic modelling accounted for one paper each. On the other hand, 18 studies focused on general scenario-based transport cost models rather than employing specific data analysis techniques (Table 4). Woo et al. (2011) argued that some techniques are employed for particular problems and topics and therefore this could explain the emergence of more purpose specific techniques (e.g. SEM, DEA, logit models) apart from descriptive statistics in port research between 1980s-2000s.

In a similar vein, it could be argued that scenario-based cost models and optimisation techniques, to a lesser extent, are prevalent in the literature because of the attention that researchers give to the investigation of the overall competitiveness of Arctic routes over the traditional ones. Thus, they have focused on specific aspects and operational problems and provided new insights and counter-arguments by capitalising on the various modelling approaches developed between 1992 and 2017. At the same time researchers from various disciplinary backgrounds have been trying to address their research questions through more sophisticated techniques. Nevertheless, Arctic shipping is an emerging topic within maritime transport research and it is expected that new techniques will emerge in the future to address specific research inquiries. Besides, it was only from 2011 that model-based techniques other than transport cost models have been employed extensively, except for two papers reporting Monte Carlo simulation between 2007 and 2009. 
Table 4. Methodological considerations of the reviewed articles

\begin{tabular}{llc}
\hline Methodological Characteristics & Categories & No. of articles \\
\hline \multirow{3}{*}{ Research Methods } & Analytical Mathematical & 27 \\
& Empirical Statistical & 4 \\
& Empirical Case Study & 2 \\
& Transport cost model & 18 \\
& Optimisation model & 4 \\
Data Analysis Techniques & Regression analysis & 3 \\
& Monte Carlo simulation & 2 \\
& Logit model & 1 \\
& Multi-Criteria Decision-Making & 1 \\
& GIS simulation & 1 \\
& Structural Economic Model & 1 \\
& None & 2 \\
\hline
\end{tabular}

\subsection{Research considerations}

This section describes the results of the review through a narrative synthesis approach. The emphasis lies in the problems investigated and areas explored in the literature. The basic attributes of the reviewed studies such as routes, transport systems, comparison mode, and period of operations as well as the origin-destination (OD) pairs are reported in Table 5.

\subsubsection{Routes, transport systems and OD pairs}

The majority of the reviewed papers undertook an assessment of the NSR (22), six studies displayed the choice of the NWP, four assessed both routes and one tackled with the feasibility of both the NSR and the TSR. Eight studies included environmental assessments based on either $\mathrm{CO}_{2}$ emissions or other relevant GHG emissions. Most of them juxtaposed Arctic routes with the Suez Canal route (27), seven of them chose the route via the Panama Canal and one considered the route via the Cape of Good Hope amongst others. Three papers reported the Trans-Siberian Railway, one an all-air route, five papers included intermodal routes (seaair/sea-rail/truck-rail) as alternatives, whereas three studies examined a combined schedule (summer season in the NSR and winter season in the Suez Canal route).

Liner shipping is the dominant transport system studied (20 studies), six studies selected dry bulk segments, three studies examined oil tanker segments, two studies dealt with liquefied natural gas (LNG) tanker shipping, and two studies investigated both liner and bulk shipping. The OD pairs vary widely in terms of the ports chosen by the identified studies. The majority of the reviewed papers focus on origins in Northwest Europe and destinations in East Asia, one included a large number of ODs whilst some others opted for ports in Russia, USA, Mexico and Canada. 
Table 5. Comparative studies between Arctic and traditional routes

\begin{tabular}{|c|c|c|c|c|c|c|c|}
\hline $\begin{array}{l}\text { Author(s) } \\
\text { and year }\end{array}$ & Title & Journal & $\begin{array}{l}\text { Comparison } \\
\text { \& Scope }\end{array}$ & $\begin{array}{l}\text { Transport } \\
\text { Systems }\end{array}$ & Routes & $\begin{array}{l}\text { Time frame of } \\
\text { operations }\end{array}$ & $\begin{array}{l}\text { Origin-Destination } \\
\text { Pairs }\end{array}$ \\
\hline $\begin{array}{l}\text { Wergeland } \\
\text { (1992) }\end{array}$ & $\begin{array}{l}\text { The northern sea route - rosy } \\
\text { prospects for commercial } \\
\text { shipping? }\end{array}$ & $\begin{array}{l}\text { International } \\
\text { Challenges }\end{array}$ & $\begin{array}{l}\text { Transport costs per } \\
\text { tonne per month in US\$ }\end{array}$ & $\begin{array}{l}\text { Liner } \\
\text { Shipping } \\
\text { (Multi- } \\
\text { purpose) }\end{array}$ & $\begin{array}{l}\text { Northern Sea Route } \\
\text { - Suez Canal \& } \\
\text { Panama Canal }\end{array}$ & $\begin{array}{l}\text { Single leg } \\
\text { voyage }\end{array}$ & $\begin{array}{l}\text { 1. Dutch Harbour - } \\
\text { Hamburg } \\
\text { 2. Hamburg - Yokohama }\end{array}$ \\
\hline $\begin{array}{l}\text { Kondo and } \\
\text { Takamasa } \\
\text { (1999) }\end{array}$ & $\begin{array}{l}\text { The economic potential of a } \\
\text { cassette-type-reactor installed } \\
\text { nuclear ice-breaking container } \\
\text { ship }\end{array}$ & $\begin{array}{l}\text { Journal of } \\
\text { Nuclear } \\
\text { Science and } \\
\text { Technology }\end{array}$ & $\begin{array}{l}\text { Transport costs per } \\
\text { TEU per year in US\$ \& } \\
\text { per 20/40-year period; } \\
\text { Total Shipper's Costs; } \\
\text { Emission taxes } \\
\text { included }\end{array}$ & $\begin{array}{l}\text { Liner } \\
\text { Shipping }\end{array}$ & $\begin{array}{l}\text { Northern Sea Route } \\
\text { - Suez Canal }\end{array}$ & $\begin{array}{l}\text { Year-round } \\
(20 \text { - and } 40 \text {-year } \\
\text { period })\end{array}$ & Hamburg - Yokohama \\
\hline Guy (2006) & $\begin{array}{l}\text { Evaluating the viability of } \\
\text { commercial shipping in the } \\
\text { Northwest Passage }\end{array}$ & $\begin{array}{l}\text { Journal of } \\
\text { Ocean } \\
\text { Technology }\end{array}$ & $\begin{array}{l}\text { Transport costs and } \\
\text { profits in US\$ }\end{array}$ & $\begin{array}{l}\text { Liner \& Bulk } \\
\text { Shipping } \\
\text { (Dry) }\end{array}$ & $\begin{array}{l}\text { Northwest Passage } \\
\text { - Suez Canal }\end{array}$ & $\begin{array}{l}\text { Single leg } \\
\text { voyage }\end{array}$ & $\begin{array}{l}\text { 1. Rotterdam - Shanghai } \\
\text { 2. Arctic region }- \text { ? }\end{array}$ \\
\hline $\begin{array}{l}\text { Somanathan } \\
\text { et al. (2007) }\end{array}$ & $\begin{array}{l}\text { Feasibility of a sea route through } \\
\text { the Canadian arctic }\end{array}$ & $\begin{array}{l}\text { Maritime } \\
\text { Economics \& } \\
\text { Logistics }\end{array}$ & $\begin{array}{l}\text { Required freight rate } \\
\text { (RFR) per TEU in US\$ }\end{array}$ & $\begin{array}{l}\text { Liner } \\
\text { Shipping }\end{array}$ & $\begin{array}{l}\text { Northwest Passage } \\
\text { - Panama Canal }\end{array}$ & Year-round & $\begin{array}{l}\text { 1. New York-Yokohama } \\
\text { 2. St. Johns, } \\
\text { Newfoundland - } \\
\text { Yokohama }\end{array}$ \\
\hline $\begin{array}{l}\text { Somanathan } \\
\text { et al. }(2009)\end{array}$ & $\begin{array}{l}\text { The Northwest Passage: A } \\
\text { simulation }\end{array}$ & $\begin{array}{l}\text { Transportation } \\
\text { Research Part } \\
\text { A: Policy and } \\
\text { Practice }\end{array}$ & $\begin{array}{l}\text { Required freight rate } \\
\text { (RFR) per TEU in US\$ }\end{array}$ & $\begin{array}{l}\text { Liner } \\
\text { Shipping }\end{array}$ & $\begin{array}{l}\text { Northwest Passage } \\
\text { - Panama Canal }\end{array}$ & Year-round & $\begin{array}{l}\text { 1. New York-Yokohama } \\
\text { 2. St. Johns, } \\
\text { Newfoundland - } \\
\text { Yokohama }\end{array}$ \\
\hline $\begin{array}{l}\text { Verny and } \\
\text { Grigentin } \\
\text { (2009) }\end{array}$ & $\begin{array}{l}\text { Container shipping on the } \\
\text { Northern Sea Route }\end{array}$ & $\begin{array}{l}\text { International } \\
\text { Journal of } \\
\text { Production } \\
\text { Economics }\end{array}$ & $\begin{array}{l}\text { Transport costs per } \\
\text { TEU in US\$ }\end{array}$ & $\begin{array}{l}\text { Liner } \\
\text { Shipping }\end{array}$ & $\begin{array}{l}\text { 1. NSR - SCR } \\
\text { 2. Trans-Siberian } \\
\text { Railway } \\
\text { 3. Sea and air } \\
\text { 4. All-air }\end{array}$ & Year-round & $\begin{array}{l}\text { 1. Hamburg - Shanghai } \\
\text { (eastbound stop at } \\
\text { Rotterdam, westbound } \\
\text { stops: Pusan, Tokyo) } \\
\text { 2. via Dubai (Sea and air) }\end{array}$ \\
\hline $\begin{array}{l}\text { Liu and } \\
\text { Kronbak } \\
(\mathbf{2 0 1 0})\end{array}$ & $\begin{array}{l}\text { The potential economic viability } \\
\text { of using the Northern Sea Route } \\
\text { (NSR) as an alternative route } \\
\text { between Asia and Europe }\end{array}$ & $\begin{array}{l}\text { Journal of } \\
\text { Transport } \\
\text { Geography }\end{array}$ & $\begin{array}{l}\text { Profits per year } \\
\text { in US\$ }\end{array}$ & $\begin{array}{l}\text { Liner } \\
\text { Shipping }\end{array}$ & $\begin{array}{l}\text { Combined use of } \\
\text { Northern Sea Route } \\
\text { and the Suez Canal } \\
\text { - Suez Canal }\end{array}$ & $\begin{array}{l}\text { 1. Year-round for SCR } \\
\text { 2. Combined: Three, } \\
\text { six and nine month } \\
\text { periods for the NSR } \\
\text { and the rest on SCR }\end{array}$ & Rotterdam - Yokohama \\
\hline $\begin{array}{l}\text { Khon and } \\
\text { Mokhov } \\
\text { (2010) }\end{array}$ & $\begin{array}{l}\text { Arctic climate changes and } \\
\text { possible conditions of Arctic } \\
\text { navigation in the } 21 \text { st century }\end{array}$ & $\begin{array}{l}\text { Izvestiya, } \\
\text { Atmospheric } \\
\text { and Oceanic } \\
\text { Physics } \\
\end{array}$ & $\begin{array}{l}\text { Transport costs per year } \\
\text { in US\$ }\end{array}$ & $\begin{array}{l}\text { Liner } \\
\text { Shipping } \\
\text { (Multi- } \\
\text { purpose) }\end{array}$ & $\begin{array}{l}\text { Northern Sea Route } \\
\text { - Suez Canal }\end{array}$ & $\begin{array}{l}\text { Year-round } \\
\text { (end of } 21 \text { st century) }\end{array}$ & $\begin{array}{l}\text { Western Europe - Far } \\
\text { East }\end{array}$ \\
\hline
\end{tabular}




\begin{tabular}{|c|c|c|c|c|c|c|c|}
\hline $\begin{array}{l}\text { Author(s) } \\
\text { and year }\end{array}$ & Title & Journal & $\begin{array}{l}\text { Comparison } \\
\text { \& Scope }\end{array}$ & $\begin{array}{l}\text { Transport } \\
\text { Systems }\end{array}$ & Routes & $\begin{array}{l}\text { Time frame of } \\
\text { operations }\end{array}$ & $\begin{array}{l}\text { Origin-Destination } \\
\text { Pairs }\end{array}$ \\
\hline $\begin{array}{l}\text { Khon et al. } \\
\text { (2010) }\end{array}$ & $\begin{array}{l}\text { Perspectives of Northern Sea } \\
\text { Route and Northwest Passage in } \\
\text { the twenty-first century }\end{array}$ & Climatic Change & $\begin{array}{l}\text { Transport costs } \\
\text { per year in US\$ }\end{array}$ & $\begin{array}{l}\text { Liner } \\
\text { Shipping } \\
\text { (Multi- } \\
\text { purpose) }\end{array}$ & $\begin{array}{l}\text { Northern Sea Route - } \\
\text { Suez Canal }\end{array}$ & $\begin{array}{l}\text { Year-round } \\
\text { (end of 21st } \\
\text { century) }\end{array}$ & $\begin{array}{l}\text { Western Europe - Far } \\
\text { East }\end{array}$ \\
\hline $\begin{array}{l}\text { Schøyen and } \\
\text { Bråthen } \\
(2011)\end{array}$ & $\begin{array}{l}\text { The Northern Sea Route versus } \\
\text { the Suez Canal: cases from bulk } \\
\text { shipping }\end{array}$ & $\begin{array}{l}\text { Journal of } \\
\text { Transport } \\
\text { Geography }\end{array}$ & $\begin{array}{l}\text { Transport costs } \\
\text { per metric tonne } \\
(\mathrm{mt}) \text { in US\$; } \\
\mathrm{CO}_{2} \text { emissions } \\
\text { assessment }\end{array}$ & $\begin{array}{l}\text { Bulk Shipping } \\
\text { (Dry) }\end{array}$ & $\begin{array}{l}\text { 1. Northern Sea Route - } \\
\text { Suez Canal \& Cape of } \\
\text { Good Hope } \\
\text { 2. NSR - SCR }\end{array}$ & Single leg voyage & $\begin{array}{l}\text { 1. Porsgrunn (Norway) - } \\
\text { Shekou (China) } \\
\text { 2. Narvik (Norway) - } \\
\text { Qingdao (China) }\end{array}$ \\
\hline $\begin{array}{l}\text { Tavasszy et al. } \\
\text { (2011) }\end{array}$ & $\begin{array}{l}\text { A strategic network choice } \\
\text { model for global container } \\
\text { flows: specification, estimation } \\
\text { and application }\end{array}$ & $\begin{array}{l}\text { Journal of } \\
\text { Transport } \\
\text { Geography }\end{array}$ & $\begin{array}{l}\text { Container flows } \\
\text { in TEU; } \\
\text { Transport costs } \\
\text { per TEU/km } \\
\text { in US\$ }\end{array}$ & & $\begin{array}{l}\text { NSR \& NWP? \& many } \\
\text { other global land- and } \\
\text { sea-based routes }\end{array}$ & Year-round & 437 ports \\
\hline $\begin{array}{l}\text { Xu et al. } \\
\text { (2011) }\end{array}$ & $\begin{array}{l}\text { The potential seasonal } \\
\text { alternative of Asia-Europe } \\
\text { container service via Northern } \\
\text { sea route under the Arctic sea } \\
\text { ice retreat }\end{array}$ & $\begin{array}{l}\text { Maritime Policy } \\
\& \text { Management }\end{array}$ & $\begin{array}{l}\text { Transport costs } \\
\text { in US\$ }\end{array}$ & $\begin{array}{l}\text { Liner } \\
\text { Shipping }\end{array}$ & $\begin{array}{l}\text { Combined use of } \\
\text { Northern Sea Route and } \\
\text { the Suez Canal } \\
\text { - Suez Canal }\end{array}$ & $\begin{array}{l}\text { 1. Year-round for } \\
\text { SCR } \\
\text { 2. Combined } \\
\text { schedule including a } \\
\text { seasonal transit via } \\
\text { the NSR }\end{array}$ & $\begin{array}{l}\text { Series of ports between } \\
\text { North-west Europe and } \\
\text { East Asia (including } \\
\text { Taiwan) }\end{array}$ \\
\hline $\begin{array}{l}\text { Fan et al. } \\
\text { (2012) }\end{array}$ & $\begin{array}{l}\text { Impacts of new routes and ports } \\
\text { on spatial competition for } \\
\text { containerized imports into the } \\
\text { United States }\end{array}$ & $\begin{array}{l}\text { Maritime Policy } \\
\& \text { Management }\end{array}$ & $\begin{array}{l}\text { Transport costs } \\
\text { per TEU } \\
\text { in US\$; } \\
\text { Container } \\
\text { Flows in TEU }\end{array}$ & $\begin{array}{l}\text { Liner } \\
\text { Shipping }\end{array}$ & $\begin{array}{l}\text { 1.Trans-Pacific to US } \\
\text { West Coast } \\
\text { 2. Panama Canal-Gulf/US } \\
\text { East Coast } \\
\text { 3. NWP - US East Coast } \\
\text { 4. Trans-Atlantic - } \\
\text { Gulf/US East Coast } \\
\text { 5. US \& Canadian Inland } \\
\text { rail/truck corridors }\end{array}$ & $\begin{array}{l}\text { Seasonal: } \\
\text { One third of the } \\
\text { year on the NWP } \\
\text { Annual for all other } \\
\text { routes }\end{array}$ & $\begin{array}{l}31 \text { US Ports } \\
\text { Canadian Ports: } \\
\text { Vancouver, Prince } \\
\text { Rupert, Halifax, } \\
\text { Montreal, Toronto } \\
\text { Mexican West Coast } \\
\text { Ports: Manzanillo, Lazaro } \\
\text { Cardenas, Punta Colonet }\end{array}$ \\
\hline $\begin{array}{l}\text { Song and } \\
\text { Zhang (2013) }\end{array}$ & $\begin{array}{l}\text { The economy analysis of sailing } \\
\text { in the arctic Northeast Passage }\end{array}$ & $\begin{array}{l}\text { Applied } \\
\text { Mechanics and } \\
\text { Materials }\end{array}$ & $\begin{array}{l}\text { Required freight } \\
\text { rate (RFR) per } \\
\text { tonne in US\$ }\end{array}$ & $\begin{array}{l}\text { Bulk Shipping } \\
\text { (Oil Tanker) }\end{array}$ & $\begin{array}{l}\text { Northern Sea Route - } \\
\text { Suez Canal }\end{array}$ & $\begin{array}{l}\text { Summer season } \\
\text { (100 days) }\end{array}$ & Murmansk - Shanghai \\
\hline $\begin{array}{l}\text { Lasserre } \\
\text { (2014) }\end{array}$ & $\begin{array}{l}\text { Case studies of shipping along } \\
\text { Arctic routes. Analysis and } \\
\text { profitability perspectives for the } \\
\text { container sector }\end{array}$ & $\begin{array}{l}\text { Transportation } \\
\text { Research Part A: } \\
\text { Policy and } \\
\text { Practice } \\
\end{array}$ & $\begin{array}{l}\text { Transport costs } \\
\text { per TEU in US\$ }\end{array}$ & $\begin{array}{l}\text { Liner } \\
\text { Shipping }\end{array}$ & $\begin{array}{l}\text { 1. Northern Sea Route - } \\
\text { Suez Canal } \\
\text { 2. Northwest Passage - } \\
\text { Suez Canal }\end{array}$ & $\begin{array}{l}\text { Summer season } \\
\text { (six months) }\end{array}$ & $\begin{array}{l}\text { 1. Rotterdam - Shanghai/ } \\
\text { Yokohama } \\
\text { 2. Rotterdam - } \\
\text { Shanghai/Yokohama }\end{array}$ \\
\hline
\end{tabular}




\begin{tabular}{|c|c|c|c|c|c|c|c|}
\hline $\begin{array}{l}\text { Author(s) } \\
\text { and year }\end{array}$ & Title & Journal & $\begin{array}{l}\text { Comparison } \\
\text { \& Scope }\end{array}$ & $\begin{array}{l}\text { Transport } \\
\text { Systems }\end{array}$ & Routes & $\begin{array}{l}\text { Time frame of } \\
\text { operations }\end{array}$ & $\begin{array}{l}\text { Origin-Destination } \\
\text { Pairs }\end{array}$ \\
\hline $\begin{array}{l}\text { Raza and } \\
\text { Schøyen } \\
(2014)\end{array}$ & $\begin{array}{l}\text { The commercial potential for } \\
\text { LNG shipping between Europe } \\
\text { and Asia via the northern sea } \\
\text { route }\end{array}$ & $\begin{array}{l}\text { Journal of Maritime } \\
\text { Research }\end{array}$ & $\begin{array}{l}\text { Transport costs } \\
\text { per tonne and } \\
\text { MMBtu in US\$ }\end{array}$ & $\begin{array}{l}\text { Specialised } \\
\text { Shipping } \\
\text { (LNG tanker) }\end{array}$ & $\begin{array}{l}\text { Northern Sea Route } \\
\text { - Suez Canal }\end{array}$ & Round voyage & $\begin{array}{l}\text { Hammerfest (Norway) - } \\
\text { Tobata (Japan) }\end{array}$ \\
\hline $\begin{array}{l}\text { Lu et al. } \\
\text { (2014) }\end{array}$ & $\begin{array}{l}\text { An Economic Analysis of } \\
\text { Container Shipping through } \\
\text { Canadian Northwest Passage }\end{array}$ & $\begin{array}{l}\text { International } \\
\text { Journal of e- } \\
\text { Navigation and } \\
\text { Maritime Economy }\end{array}$ & $\begin{array}{l}\text { Transport costs } \\
\text { in US\$ }\end{array}$ & $\begin{array}{l}\text { Liner } \\
\text { Shipping }\end{array}$ & $\begin{array}{l}\text { Northwest Passage } \\
\text { - Panama Canal }\end{array}$ & $\begin{array}{l}\text { Single leg } \\
\text { voyage }\end{array}$ & New York - Busan \\
\hline $\begin{array}{l}\text { Lasserre } \\
\text { (2015) }\end{array}$ & $\begin{array}{l}\text { Simulations of shipping along } \\
\text { Arctic routes: comparison, } \\
\text { analysis and economic } \\
\text { perspectives }\end{array}$ & Polar Record & $\begin{array}{l}\text { Transport costs } \\
\text { per TEU in US\$ }\end{array}$ & $\begin{array}{l}\text { Liner } \\
\text { Shipping }\end{array}$ & $\begin{array}{l}\text { 1. Northern Sea } \\
\text { Route - Suez Canal } \\
\text { 2. Northwest } \\
\text { Passage - Suez } \\
\text { Canal }\end{array}$ & $\begin{array}{l}\text { Summer season } \\
\text { (six months) } \\
\& \text { Year-round }\end{array}$ & $\begin{array}{l}\text { 1. Rotterdam - Shanghai \& } \\
\text { Rotterdam - Yokohama } \\
\text { (Both NSR and NWP) } \\
\text { 2. Rotterdam - Yokohama } \\
\text { (Year-round, NSR, NWP) }\end{array}$ \\
\hline $\begin{array}{l}\text { Furuichi and } \\
\text { Otsuka } \\
\text { (2015) }\end{array}$ & $\begin{array}{l}\text { Proposing a common platform } \\
\text { of shipping cost analysis of the } \\
\text { Northern Sea Route and the } \\
\text { Suez Canal Route }\end{array}$ & $\begin{array}{l}\text { Maritime } \\
\text { Economics \& } \\
\text { Logistics }\end{array}$ & $\begin{array}{l}\text { Transport costs } \\
\text { per TEU in US\$; } \\
\mathrm{CO}_{2} \text { emissions } \\
\text { assessment }\end{array}$ & $\begin{array}{l}\text { Liner } \\
\text { Shipping }\end{array}$ & $\begin{array}{l}\text { Combined use of } \\
\text { Northern Sea Route } \\
\text { and the Suez Canal } \\
\text { - Suez Canal }\end{array}$ & $\begin{array}{l}\text { 1. Year-round for } \\
\text { SCR } \\
\text { 2. Combined: 105; } \\
\text { 135; 165; 195; 225 } \\
\text { days in NSR and the } \\
\text { rest on SCR }\end{array}$ & Hamburg - Yokohama \\
\hline $\begin{array}{l}\text { Moon et al. } \\
\text { (2015) }\end{array}$ & $\begin{array}{l}\text { A study on competitiveness of } \\
\text { sea transport by comparing } \\
\text { international transport routes } \\
\text { between Korea and EU }\end{array}$ & $\begin{array}{l}\text { The Asian Journal } \\
\text { of Shipping and } \\
\text { Logistics }\end{array}$ & $\begin{array}{l}\text { Transport costs } \\
\text { per TEU in US\$ } \\
\text { Other quantitative } \\
\text { and qualitative } \\
\text { factors }\end{array}$ & $\begin{array}{l}\text { Liner } \\
\text { Shipping }\end{array}$ & $\begin{array}{l}\text { Trans-Korean } \\
\text { Railway/Trans- } \\
\text { Siberian Railway - } \\
\text { NSR - SCR }\end{array}$ & Single leg voyage & Busan - Berlin \\
\hline $\begin{array}{l}\text { Fan et al. } \\
(2015)\end{array}$ & $\begin{array}{l}\text { Risk analysis in port } \\
\text { competition for containerized } \\
\text { imports }\end{array}$ & $\begin{array}{l}\text { European Journal of } \\
\text { Operational } \\
\text { Research }\end{array}$ & $\begin{array}{l}\text { Container import } \\
\text { flows; } \\
\text { Transport costs } \\
\text { per TEU in US\$ }\end{array}$ & $\begin{array}{l}\text { Liner } \\
\text { Shipping }\end{array}$ & $\begin{array}{l}\text { Same as in } \\
\text { Fan et al. (2012) }\end{array}$ & Year-round? & Same as in Fan et al. (2012) \\
\hline $\begin{array}{l}\text { Chou et al. } \\
(2015)\end{array}$ & $\begin{array}{l}\text { The impact on the operation } \\
\text { costs of bulk ship after the } \\
\text { opening of the arctic route }\end{array}$ & $\begin{array}{l}\text { Advanced Science } \\
\text { Letters }\end{array}$ & $\begin{array}{l}\text { Transport costs } \\
\text { in US\$ }\end{array}$ & $\begin{array}{l}\text { Bulk } \\
\text { Shipping } \\
\text { (Dry) }\end{array}$ & $\begin{array}{l}\text { Northern Sea Route } \\
\text { - Suez Canal }\end{array}$ & Single leg voyage & $\begin{array}{l}\text { Kaohsiung/Keelung/ } \\
\text { Shanghai/Busan - } \\
\text { Rotterdam }\end{array}$ \\
\hline $\begin{array}{l}\text { Chang et al. } \\
\text { (2015) }\end{array}$ & $\begin{array}{l}\text { Route planning and cost } \\
\text { analysis for travelling through } \\
\text { the Arctic Northeast Passage } \\
\text { using public 3D GIS }\end{array}$ & $\begin{array}{l}\text { International } \\
\text { Journal of } \\
\text { Geographical } \\
\text { Information Science }\end{array}$ & $\begin{array}{l}\text { Transport costs } \\
\text { in US\$ }\end{array}$ & $\begin{array}{l}\text { Bulk Shipping } \\
\text { (Dry) }\end{array}$ & $\begin{array}{l}\text { Northern Sea Route } \\
\text { - Suez Canal }\end{array}$ & Single leg voyage & $\begin{array}{l}\text { Series of ports between } \\
\text { North-western Europe and } \\
\text { Northeast Asia }\end{array}$ \\
\hline $\begin{array}{l}\text { Cariou and } \\
\text { Faury } \\
(2015)\end{array}$ & $\begin{array}{l}\text { Relevance of the Northern Sea } \\
\text { Route (NSR) for bulk shipping }\end{array}$ & $\begin{array}{l}\text { Transportation } \\
\text { Research Part A: } \\
\text { Policy and Practice }\end{array}$ & $\begin{array}{l}\text { Transport costs } \\
\text { in US\$; Carbon } \\
\text { tax included }\end{array}$ & $\begin{array}{l}\text { Bulk Shipping } \\
\text { (Dry) }\end{array}$ & $\begin{array}{l}\text { Northern Sea Route } \\
\text { - Suez Canal }\end{array}$ & Single leg voyage & $\begin{array}{l}\text { Porsgrunn (Norway) - } \\
\text { Shekou (China) }\end{array}$ \\
\hline
\end{tabular}




\begin{tabular}{|c|c|c|c|c|c|c|c|}
\hline $\begin{array}{l}\text { Author(s) } \\
\text { and year }\end{array}$ & Title & Journal & $\begin{array}{l}\text { Comparison } \\
\text { \& Scope }\end{array}$ & $\begin{array}{l}\text { Transport } \\
\text { Systems }\end{array}$ & Routes & $\begin{array}{l}\text { Time frame } \\
\text { of operations }\end{array}$ & $\begin{array}{l}\text { Origin-Destination } \\
\text { Pairs }\end{array}$ \\
\hline $\begin{array}{l}\text { Lindstad et al. } \\
\text { (2016) }\end{array}$ & $\begin{array}{l}\text { Economic savings linked to } \\
\text { future Arctic shipping trade } \\
\text { are at odds with climate } \\
\text { change mitigation }\end{array}$ & Transport Policy & $\begin{array}{l}\text { Transport costs per } \\
\text { tonne in US\$; } \\
\text { Emissions assessment }\end{array}$ & $\begin{array}{l}\text { Bulk Shipping } \\
\text { (Dry) }\end{array}$ & $\begin{array}{l}\text { Northern Sea Route } \\
\text { - Suez Canal }\end{array}$ & N.A. & $\begin{array}{l}\text { Asia - Europe (Not } \\
\text { specified ports) }\end{array}$ \\
\hline $\begin{array}{l}\text { Pruyn } \\
(2016)\end{array}$ & $\begin{array}{l}\text { Will the Northern Sea Route } \\
\text { ever be a viable alternative? }\end{array}$ & $\begin{array}{l}\text { Maritime Policy \& } \\
\text { Management }\end{array}$ & $\begin{array}{l}\text { Transport } \\
\text { costs/charter rates per } \\
\text { tonne in US\$ }\end{array}$ & $\begin{array}{l}\text { Bulk Shipping } \\
\text { (Dry) }\end{array}$ & $\begin{array}{l}\text { Northern Sea Route } \\
\text { - Suez Canal }\end{array}$ & Year-round & $\begin{array}{l}\text { Baltic/Hamburg and Le } \\
\text { Havre Range - } \\
\text { China/Southeast Asia }\end{array}$ \\
\hline $\begin{array}{l}\text { Zhao et al. } \\
\text { (2016) }\end{array}$ & $\begin{array}{l}\text { Study on China-EU container } \\
\text { shipping network in the } \\
\text { context of Northern Sea } \\
\text { Route }\end{array}$ & $\begin{array}{l}\text { Journal of Transport } \\
\text { Geography }\end{array}$ & $\begin{array}{l}\text { Profits per year } \\
\text { in US\$ }\end{array}$ & $\begin{array}{l}\text { Liner } \\
\text { Shipping }\end{array}$ & $\begin{array}{l}\text { Northern Sea Route } \\
\text { - Suez Canal }\end{array}$ & Year-round & $\begin{array}{l}\text { Series of ports between } \\
\text { North-western Europe } \\
\text { (including Spain) and } \\
\text { Northeast Asia }\end{array}$ \\
\hline $\begin{array}{l}\text { Zhao and Hu } \\
\text { (2016) }\end{array}$ & $\begin{array}{l}\text { Study on economic } \\
\text { evaluation of the northern sea } \\
\text { route: taking the voyage of } \\
\text { Yong Sheng as an example }\end{array}$ & $\begin{array}{l}\text { Transportation } \\
\text { Research Board }\end{array}$ & $\begin{array}{l}\text { Transport costs } \\
\text { in US\$; } \\
\text { Emissions assessment }\end{array}$ & $\begin{array}{l}\text { Liner } \\
\text { Shipping } \\
\text { (Multi- } \\
\text { purpose) }\end{array}$ & $\begin{array}{l}\text { Northern Sea Route } \\
\text { - Suez Canal }\end{array}$ & $\begin{array}{l}\text { Year-round \& } \\
\text { Single leg voyage }\end{array}$ & $\begin{array}{l}\text { Taicang (China) - } \\
\text { Rotterdam (The } \\
\text { Netherlands) }\end{array}$ \\
\hline $\begin{array}{l}\text { Zhang et al. } \\
\text { (2016) }\end{array}$ & $\begin{array}{l}\text { Shipping efficiency } \\
\text { comparison between northern } \\
\text { sea route and the } \\
\text { conventional Asia-Europe } \\
\text { shipping route via Suez Canal }\end{array}$ & $\begin{array}{l}\text { Journal of Transport } \\
\text { Geography }\end{array}$ & $\begin{array}{l}\text { Transport costs per } \\
\text { tonne in US\$ and } \\
\text { profits per TEU in } \\
\text { US\$ }\end{array}$ & $\begin{array}{l}\text { Liner \& Bulk } \\
\text { Shipping (Oil } \\
\text { Tanker) }\end{array}$ & $\begin{array}{l}\text { Northern Sea Route } \\
\text { - Suez Canal }\end{array}$ & $\begin{array}{l}\text { Round voyage for } \\
\text { both cases }\end{array}$ & $\begin{array}{l}\text { Liner Shipping: Shanghai } \\
\text { - Rotterdam (including } \\
\text { stopovers) Bulk Shipping: } \\
\text { Mongstad (Norway) - } \\
\text { Mizushima (Japan) }\end{array}$ \\
\hline $\begin{array}{l}\text { Faury and } \\
\text { Cariou } \\
(2016)\end{array}$ & $\begin{array}{l}\text { The Northern Sea Route } \\
\text { competitiveness for oil } \\
\text { tankers }\end{array}$ & $\begin{array}{l}\text { Transportation } \\
\text { Research Part A: } \\
\text { Policy and Practice }\end{array}$ & $\begin{array}{l}\text { Transport costs } \\
\text { in US\$ and transit } \\
\text { time per month }\end{array}$ & $\begin{array}{l}\text { Bulk Shipping } \\
\text { (Oil Tanker) }\end{array}$ & $\begin{array}{l}\text { Northern Sea Route } \\
\text { - Suez Canal }\end{array}$ & $\begin{array}{l}\text { June - February } \\
\text { (Lower Bound) } \\
\text { July - December } \\
\text { (Higher Bound) }\end{array}$ & $\begin{array}{l}\text { Murmansk (Russia) - } \\
\text { Daesan (South Korea) }\end{array}$ \\
\hline $\begin{array}{l}\text { Wang et al. } \\
2016\end{array}$ & $\begin{array}{l}\text { Comments on "Case studies } \\
\text { of shipping along Arctic } \\
\text { routes. Analysis and } \\
\text { profitability perspectives for } \\
\text { the container sector" }\end{array}$ & $\begin{array}{l}\text { Transportation } \\
\text { Research Part A: } \\
\text { Policy and Practice }\end{array}$ & $\begin{array}{l}\text { Transport costs per } \\
\text { TEU in US\$ } \\
\text { (In-transit inventory } \\
\text { costs included) }\end{array}$ & $\begin{array}{l}\text { Liner } \\
\text { Shipping }\end{array}$ & $\begin{array}{l}\text { 1. Northern Sea } \\
\text { Route - Suez Canal } \\
\text { 2. Northwest } \\
\text { Passage - Suez } \\
\text { Canal }\end{array}$ & $\begin{array}{l}\text { Summer season } \\
\text { (six months) }\end{array}$ & $\begin{array}{l}\text { 1. Rotterdam - } \\
\text { Shanghai/Yokohama } \\
\text { 2. Rotterdam - Shanghai/ } \\
\text { Yokohama }\end{array}$ \\
\hline $\begin{array}{l}\text { Chou et al. } \\
(2017)\end{array}$ & $\begin{array}{l}\text { Fuel consumption ratio } \\
\text { analysis for transiting from } \\
\text { various ports and harbours in } \\
\text { Asia through the Northern } \\
\text { Sea Route }\end{array}$ & $\begin{array}{l}\text { The Journal of } \\
\text { Navigation }\end{array}$ & $\begin{array}{l}\text { Fuel consumption } \\
\text { per TEU; } \\
\mathrm{CO}_{2} \text { emissions } \\
\text { per TEU }\end{array}$ & $\begin{array}{l}\text { Liner } \\
\text { Shipping }\end{array}$ & $\begin{array}{l}\text { Northern Sea Route } \\
\text { - Suez Canal }\end{array}$ & Single leg voyage & $\begin{array}{l}\text { Rotterdam - } \\
\text { Busan/Yokohama/ } \\
\text { Shanghai/Kaoshiung/ } \\
\text { Hong Kong/ Singapore }\end{array}$ \\
\hline $\begin{array}{l}\text { Schröder et al. } \\
\text { (2017) }\end{array}$ & $\begin{array}{l}\text { Environmental impact of } \\
\text { exhaust emissions by Arctic } \\
\text { Shipping }\end{array}$ & Ambio & $\begin{array}{l}\text { Fuel consumption } \\
\text { in } \mathrm{t} / \text { day } \\
\text { Emissions in } \\
\mathrm{kg} / \text { voyage }\end{array}$ & $\begin{array}{l}\text { Bulk \& } \\
\text { Specialised } \\
\text { Shipping (Oil } \\
\text { \& LNG } \\
\text { Tanker) } \\
\end{array}$ & $\begin{array}{l}\text { NSR (Three sub- } \\
\text { routes) - TSR - } \\
\text { SCR }\end{array}$ & $\begin{array}{l}\text { Single leg voyage } \\
\text { April/July/ } \\
\text { September/ } \\
\text { November } \\
\text { 1960/2000/2040 }\end{array}$ & Rotterdam - Yokohama \\
\hline
\end{tabular}




\subsubsection{Cost, operational and revenue factors}

A breakdown of the cost structure according to Stopford's (2009) cash flow model and other operational characteristics was further attempted in order to expound the assumptions, based on which these studies determined the competitiveness of Arctic routes (Appendix A). The operating costs discerned from the reviewed papers are crew wages, insurance, and repair and maintenance. The voyage costs or factors which affect them are fuel consumption, speed, bunker fuel prices and transit fees. Further, capital costs were reported, including additional expenses (premium) required for an ice-class vessel (vessel equipped with an enhanced hull and other arrangements to be able to sail in icy waters). The ship revenue factors are operating speed, ship size and ice-class and dwt utilisation (Stopford, 2009, p. 220).

Five studies merely report additional operating costs for either the NSR or the NWP without providing a more detailed analysis on the specific elements of these costs. Guy (2006) assumed trip charter premiums between $15-200 \%$, reflecting increased operating and capital costs of an ice-strengthened vessel. In a similar vein, Schøyen and Bråthen (2011) asserted that when considering Arctic maritime operations in the NSR, then a $20 \%$ premium should be applied in the usual operating expenses whilst Zhang et al. (2016) and Faury and Cariou (2016) used the same premium for oil tankers in their study.

Increased crew costs range from 10\% (Liu and Kronbak, 2010; Lasserre, 2014; Zhao et al., 2016; Zhang et al., 2016) to 28\% (Song and Zhang, 2013). Five studies did not include crew costs (Xu et al., 2011; Raza and Schøyen, 2014; Lu et al., 2014; Chang et al., 2015; Pruyn et al., 2016). It is possible that most of these studies assumed that manning costs are same regardless of the route, whilst others considered differences depending on the route but these could not be discerned from their assumptions. Higher repair and maintenance costs were assumed at a range of $20 \%$ (Zhang et al., 2016) to 100\% (Verny and Grigentin, 2009, Liu and Kronbak, 2010) in most of the cases.

Most of the papers incorporated insurance premiums related to Hull and Machinery (H\&M), and Protection and Indemnity (P\&I) insurance. The insurance premiums discerned from the review range from 5\% (Song and Zhang, 2013) to 50-80\% (Lasserre, 2015) concerning both the H\&M and P\&I. Pruyn (2016) assumed a 100\% premium for P\&I and a 200\% premium for H\&M whereas Zhao et al. (2016) and Zhang et al. (2016) included 25\% premium for P\&I and $100 \%$ and $50 \%$ for H\&M respectively in the case of ice class containerships. Xu et al. (2011) assumed a NSR with less prevalent ice conditions and hence they went for non-ice class vessels and same insurance costs in both routes. Furuichi and Otsuka (2014) adopted a premium of 10 US\$/GT per year and a piracy premium of 40 US\$ per TEU.

Five of the studies referring to the NWP did not include any ice breaking fees (Somanathan et al., 2007; 2009; Lasserre, 2014; 2015; Lu et al., 2014). Five studies referring to the NSR assumed discounted fees which are in accordance with some references from shipping operators: they range from 3 US\$/tonne (ballast) to $6.8 \mathrm{US} \$ /$ tonne (laden) (Raza and Schøyen, 2014), 5 US\$/GT (Furuichi and Otsuka, 2014; Zhang et al., 2016) and 8.2 US\$/tonne (Lasserre, 2015). Nine of them consider the official NSR administration (NSRA) tariffs in their models. Pruyn (2016) assumed a scenario of no fees and a range of 4-19 US\$/tonne when fees are applied, whereas Zhao et al. (2016) assumed three scenarios based on historical data.

The average vessel speed reported differs across the papers and depends on various assumptions regarding the speed on ice, the time frame of operations and the scheduling between rival routes. Some papers emphasised on a lower speed on ice waters and a higher one on open 
waters. The speed on ice-infested waters range from 6-12 kts (Chang et al., 2015) to $14 \mathrm{kts}$ (Pruyn, 2016) for bulkers and tankers and from 3-4 kts (Fan et al., 2012) to 17.7 kts (Lasserre, 2014; 2015) for containerships.

Some of the studies hypothesised the effect either of the ice resistance or of the increased weight of an ice-class vessel on fuel consumption and therefore assumed increased rates for the Arctic routes: For containerships, they range from 8-15\% (Lasserre, 2015) to 50-58\% (Somanathan et al., 2009) and 10\% increased specific fuel oil consumption (Furuichi and Otsuka, 2015). For bulk carriers and oil tankers, they range from 5\% (Cariou and Faury, 2015, Pruyn, 2016, Faury and Cariou, 2016) to 30\% (Zhang et al., 2016). Most of the studies included a capital premium in their analyses, ranging from 5\% (Song and Zhang, 2013) to 35\% (Wergeland, 1992). Three papers assumed no premium whilst it was not discernible in the rest.

Five papers considered the costs of periodic maintenance, that is, the undertaking of regular surveys and dry docking of a vessel instead of routine repairs and maintenance which constitute part of the operating costs, ranging from 20\% to 150\% (Somanathan et al., 2007, 2009, Lasserre, 2014, 2015). Load factors were also reported for both westbound and eastbound cargoes. These factors range from $30 \%$ to $100 \%$ with some of the studies assuming lower load factors for eastbound cargoes, taking into account the trade flows between Europe and Asia.

The reviewed papers were categorised further according to the results of the Arctic routes' economic feasibility and emissions assessment (Table 6). 31 papers considered either costs/profits or emissions whereas two focused solely on emissions. Thirteen papers consider the Arctic routes either cost-competitive or profitable whilst five studies project their costeffectiveness in the long-term. Somanathan et al. (2007; 2009) and Lasserre $(2014 ; 2015)$ asserted that only specific trades are profitable whereas six studies considered the Arctic routes unprofitable or not cost-competitive. Guy (2006) found that the NWP is cost-competitive under specific scenarios whilst Faury and Cariou (2016) found the NSR to be cost-competitive in specific months of the year. Of the eight studies that appraised emissions, one concluded that Arctic routes are less energy efficient than the traditional ones (Lindstad et al., 2016).

The results listed in Table 6 make reference to the basic scenarios of the reviewed studies. The various discrepancies make the cross-comparison of the results rather difficult, especially the differences in the assumed time frame of operations. Thus, the reviewed papers were clustered by taking into account this critical factor (Figure 2).

The NSR and/or the NWP are shown not to be competitive in five out of nine papers that consider year-round operations, whilst two report mixed results. The costs using the NSR could be as high as $35.7 \%$ (Verny and Grigentin, 2009) compared to the Suez Canal route whilst the required freight rate (RFR) in NWP could be as higher as $13.4 \%$ than in the Panama Canal route (Somanathan et al., 2009).

When it comes to papers that considered a seasonal navigation period, the costs in the NSR could be $41 \%$ higher than those on the Suez Canal route. On the other hand, the NWP could be more competitive by $19.5 \%$ on the Rotterdam-Yokohama route but less competitive by $12 \%$ on the Rotterdam-Shanghai route than the Suez Canal (Lasserre, 2014; 2015). Wang et al. (2016) argued that if the time value of cargo is included in Lasserre's (2014) calculations, then both the NSR and NWP are more competitive than the Suez route by $3 \%$ and $65 \%$ respectively on the Rotterdam-Shanghai route and $52 \%$ and $85 \%$ on the Rotterdam-Yokohama route respectively. 
Table 6. Cost and emissions assessment results of the reviewed articles

\begin{tabular}{|c|c|c|c|c|c|}
\hline $\begin{array}{l}\text { Author(s) } \\
\text { and year }\end{array}$ & $\begin{array}{l}\text { Arctic } \\
\text { Route(s) }\end{array}$ & Competitive & $\begin{array}{l}\text { Not } \\
\text { Competitive }\end{array}$ & $\begin{array}{l}\text { Include } \\
\text { emissions } \\
\text { assessment }\end{array}$ & $\begin{array}{l}\text { Do not } \\
\text { include } \\
\text { emissions } \\
\text { assessment }\end{array}$ \\
\hline Wergeland (1992) & NSR & • & & & - \\
\hline $\begin{array}{l}\text { Kondo and Takamasa } \\
\text { (1999) }\end{array}$ & NSR & $\begin{array}{l}\text { Total shipper's } \\
\text { cost depending } \\
\text { on time value } \\
\text { of freight }\end{array}$ & $\begin{array}{l}\text { First year } \\
\text { transport cost \& } \\
\text { 20/40-year RFR }\end{array}$ & • & \\
\hline Guy (2006) & NWP & $\begin{array}{l}\text { Optimistic } \\
\text { Scenarios }\end{array}$ & $\begin{array}{l}\text { Pessimistic } \\
\text { Scenarios }\end{array}$ & & $\bullet$ \\
\hline $\begin{array}{l}\text { Somanathan et al. } \\
(2007)\end{array}$ & NWP & $\begin{array}{l}\text { Yokohama - } \\
\text { St. John's }\end{array}$ & $\begin{array}{l}\text { Yokohama-- } \\
\text { New York }\end{array}$ & & - \\
\hline $\begin{array}{l}\text { Somanathan et al. } \\
\text { (2009) }\end{array}$ & NWP & $\begin{array}{l}\text { Yokohama - } \\
\text { St. John's }\end{array}$ & $\begin{array}{l}\text { Yokohama - } \\
\text { New York }\end{array}$ & & $\bullet$ \\
\hline $\begin{array}{l}\text { Verny and Grigentin } \\
\text { (2009) }\end{array}$ & NSR & & - & & • \\
\hline $\begin{array}{l}\text { Liu and Kronbak } \\
\text { (2010) }\end{array}$ & NSR & & • & & • \\
\hline $\begin{array}{l}\text { Khon and Mokhov } \\
\text { (2010) }\end{array}$ & NSR & 2080-2099 & & & • \\
\hline Khon et al. (2010) & NSR & 2080-2099 & & & $\bullet$ \\
\hline $\begin{array}{l}\text { Schøyen and Bråthen } \\
\text { (2011) }\end{array}$ & NSR & • & & $\bullet$ & \\
\hline Tavasszy et al. (2011) & NSR \& NWP & Long-term & & & $\bullet$ \\
\hline Xu et al. (2011) & NSR & $\bullet$ & & & $\bullet$ \\
\hline Fan et al. (2012) & NWP & Long-term & & & $\bullet$ \\
\hline $\begin{array}{l}\text { Song and Zhang } \\
\text { (2013) }\end{array}$ & NSR & • & & & $\bullet$ \\
\hline $\begin{array}{l}\text { Lasserre } \\
(2014)\end{array}$ & NSR \& NWP & $\begin{array}{l}\text { NSR \& NWP: } \\
\text { Yokohama }\end{array}$ & $\begin{array}{l}\text { NSR: Shanghai \& } \\
\text { Yokohama } \\
\text { NWP: Shanghai }\end{array}$ & & $\bullet$ \\
\hline $\begin{array}{l}\text { Raza and Schøyen } \\
\text { (2014) }\end{array}$ & NSR & $\bullet$ & & & $\bullet$ \\
\hline Lu et al. (2014) & NWP & $\bullet$ & & & $\bullet$ \\
\hline $\begin{array}{l}\text { Lasserre } \\
(2015)\end{array}$ & NSR \& NWP & $\begin{array}{l}\text { NWP Rotterdam } \\
\text {-Yokohama }\end{array}$ & $\begin{array}{l}\text { NSR \& NWP in } \\
\text { four basic } \\
\text { scenarios }\end{array}$ & & - \\
\hline $\begin{array}{l}\text { Furuichi and Otsuka } \\
\text { (2015) }\end{array}$ & NSR & • & & • & \\
\hline Moon et al. (2015) & NSR & & $\bullet$ & & $\bullet$ \\
\hline Fan et al. (2015) & NWP & Long-term & & & $\bullet$ \\
\hline Chou et al. (2015) & NSR & $\bullet$ & & & $\bullet$ \\
\hline Chang et al. (2015) & NSR & $\bullet$ & & & $\bullet$ \\
\hline $\begin{array}{l}\text { Cariou and Faury } \\
(2015)\end{array}$ & NSR & • & & • & \\
\hline Lindstad et al. (2016) & NSR & - & & • & \\
\hline Pruyn (2016) & NSR & & $\bullet$ & & $\bullet$ \\
\hline Zhao et al. (2016) & NSR & & $\bullet$ & & $\bullet$ \\
\hline Zhao and Hu (2016) & NSR & $\bullet$ & & $\bullet$ & \\
\hline Zhang et al. (2016) & NSR & & • & & $\bullet$ \\
\hline $\begin{array}{l}\text { Faury and Cariou } \\
\text { (2016) }\end{array}$ & NSR & $\begin{array}{l}\text { July/August - } \\
\text { November }\end{array}$ & $\begin{array}{l}\text { December - } \\
\text { June }\end{array}$ & & $\bullet$ \\
\hline Wang et al. (2016) & NSR \& NWP & - & & & $\bullet$ \\
\hline Chou et al. (2017) & NSR & & & • & \\
\hline Schröder et al. (2017) & NSR \& TSR & & & $\bullet$ & \\
\hline
\end{tabular}


The Arctic routes are shown to be competitive in almost all of the papers considering single/round voyages as well as in Lindstad et al. (2016), who did not explicitly define the time frame. The competitiveness of the NSR ranges from $0.5 \%$ (Schøyen and Bråthen, 2011) to 47.2\% Chang et al. (2015). The NWP is more competitive by $4.3-32.5 \%$ for liner shipping than the Suez Canal route under the optimistic scenarios (Guy, 2006). On the other hand, the Suez Canal route could be 1-66\% more competitive than the NWP under the pessimistic scenarios (Guy, 2016).

Five papers consider Arctic routes cost-competitiveness in the long-term with two assuming a positive relationship between sea ice extent and seasonal freight rates by 2080-2099 (Khon and Mokhov, 2010; Khon et al., 2010) and three predicting future cargo flows through the Arctic based on transport costs of alternative routes (Tavasszy et al., 2011; Fan et al., 2012; 2015). On the other hand, the RFR for nuclear-powered ice-breaking ships using the NSR could be 3\% (40 years) and 10\% (20 years) higher than that on the Suez Canal route but could be $70 \%$ higher (20 years) when considering large vessels on the Suez Canal route (Kondo and Takamasa, 1999).

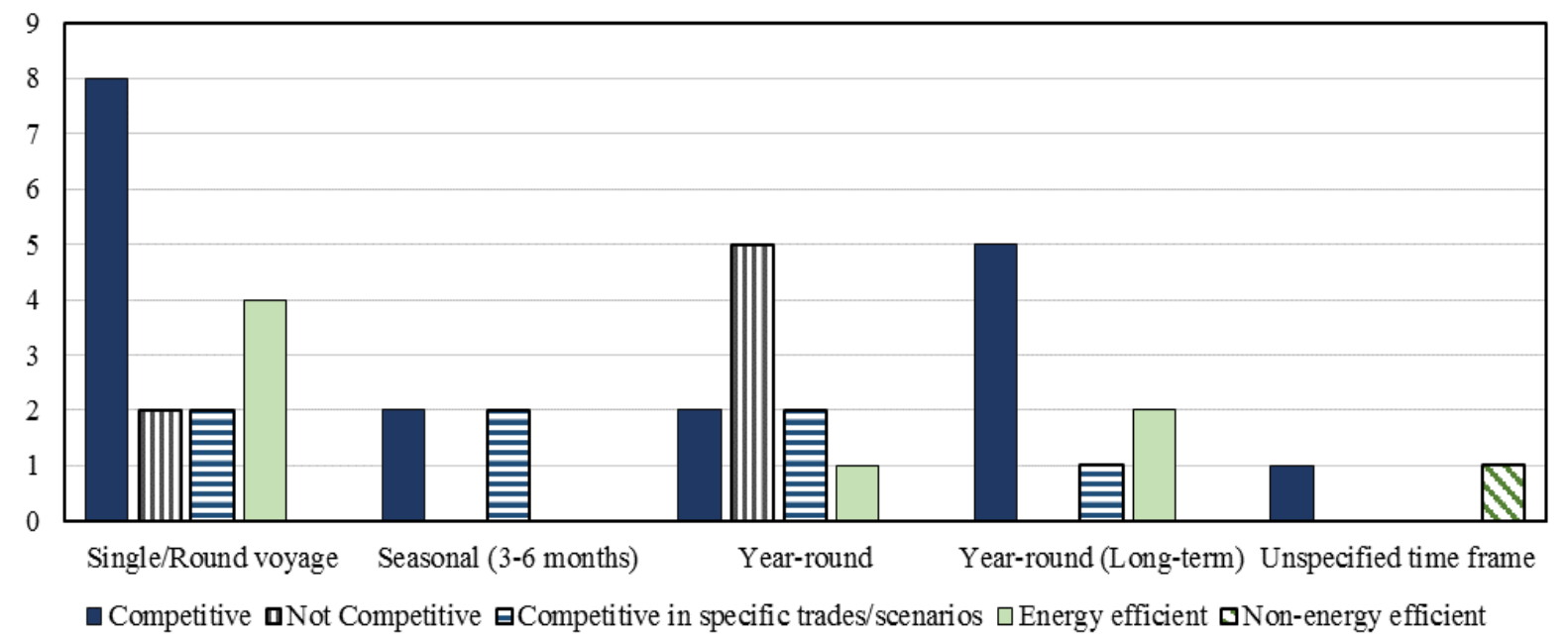

Fig. 2. Cost and emissions assessment results based on the time frame of operations

Overall, Arctic routes were found to be either cost-competitive or profitable in $42 \%$ of the studies and unprofitable or not cost-competitive in 19\%.23\% suggest that they are competitive under specific scenarios and certain trade routes. The remaining $16 \%$ of reviewed papers project that Arctic routes would become cost-effective in the long-term. It is clear from this analysis that, in most of the studies which assume an annual operating period, Arctic routes tend to be either uncompetitive or demonstrate mixed results, especially for liner shipping (e.g. Verny and Grigentin, 2009, Liu and Kronbak, 2010, Lasserre, 2015; Zhao et al., 2016). The picture is similar regarding seasonal sailings (three to six months), where two studies report mixed results in certain OD pairs under liner shipping scenarios (Lasserre 2014, 2015). In contrast, they were found competitive in most of the studies that applied round or single voyages mainly for bulk or specialised shipping (e.g. Wergeland, 1992, Schøyen and Bråthen, 2011; Raza and Schøyen, 2014, Lu et al., 2014, Cariou and Faury, 2015). Finally, the competitiveness of these routes increases for year-round liner shipping operations only in the long-term (Khon and Mokhov, 2010; Khon et al., 2010; Tavasszy et al., 2011; Fan et al., 2012; 2015). A cross-comparison of the various cost components of each study is infeasible due to lack of data and a mismatch on the available variables regarding costs or operational factors. 


\section{Discussion and future directions}

\subsection{Research insights}

The number of influential factors, which determine maritime route choice, extend to include operational, navigational, cost and revenue factors amongst others. Important variables are now discussed in order to understand how they affect route choice at the operational and tactical level, as well as the interrelations between them.

\subsubsection{Route selection}

The systematic review has identified a specific preference for simulating shipping scenarios in the NSR in most of the cases. This is not surprising if we were to consider prevailing sea ice conditions and recent infrastructure and project developments in the Arctic region, favouring mainly the NSR amongst others. However, studies within climate science investigating the future accessibility of Arctic routes indicate extended navigation seasons for several vessel types and for all the routes (NSR, NWP and TSR) throughout the 21 st century. Khon et al. (2010) estimated the navigation season to be 3-6 (2-4) months for the NSR and NWP respectively regarding low ice class ships by 2080-2099. Stephenson et al. (2013) projected the seasonal navigation period in the NSR to be approximately 103, 113 and 120 days for non-ice class, PC6 and PC3 ships respectively by the end of 21st century. Most recently, Khon et al. (2017) projected a longer navigation season for the NSR than that in Khon et al. (2010) for non-ice class ships: 4-6.5 months by late-century.

Smith and Stephenson (2013) estimated the probability of non-ice class ships to transit the NSR using September as a benchmark to be $94-98 \%$ and that on the NWP to be 53-60\% by 20402059. Stephenson et al. (2014) found high inter-annual variability of the NSR navigation season taking into account sea ice and bathymetry from 2013-2027. Further, Stephenson and Smith (2015) identified a gradual increase in the number of voyages through the TSR for PC6 vessels by mid-century whilst the possibilities of utilising the NWP rise by 2060 . In contrast, Laliberte et al. (2016) found both the NWP and TSR to be ice-covered beyond mid-century whereas regions along the NSR and Arctic Bridge are projected to be more accessible for non-ice class vessels. Their results are in line with Pizzolato et al. (2016) and Liu et al. (2017) who concluded that multi-year ice in the northern sub-route of the NWP to be a significant obstacle for shipping activities in the medium-term. According to Melia et al. (2016), the TSR is projected to become available for non-ice class ships by mid-century whilst voyages from Europe to the Far East will take 17 days by late-century. Aksenov et al. (2017) identified sea ice extent and thickness as the most determining factors for shipping in the Arctic until 2030-2050, whereas other ice properties (e.g. ice ridging, drift ice, and internal pressure), ocean circulation, winds, currents and waves will mostly affect navigation beyond that period.

Taking into account these findings, future research should also pay attention to the TSR as an alternative route. In addition, more focus is needed in simulating the NWP, and alternative subroutes of the NSR, as these could enable the employment of larger vessels. Moreover, the possible opening of the Nicaragua and Kra Canals, the expansion of both Panama and Suez Canals as well as alternative land-based (e.g. Tran-Siberian Railway, New Eurasian Land Bridges) and other established trade routes will also have an impact to their northern rivals (Fig. 3) (Tavasszy et al., 2011, Yip and Wong, 2016; Martinez et al., 2016; Zeng et al., 2017). 


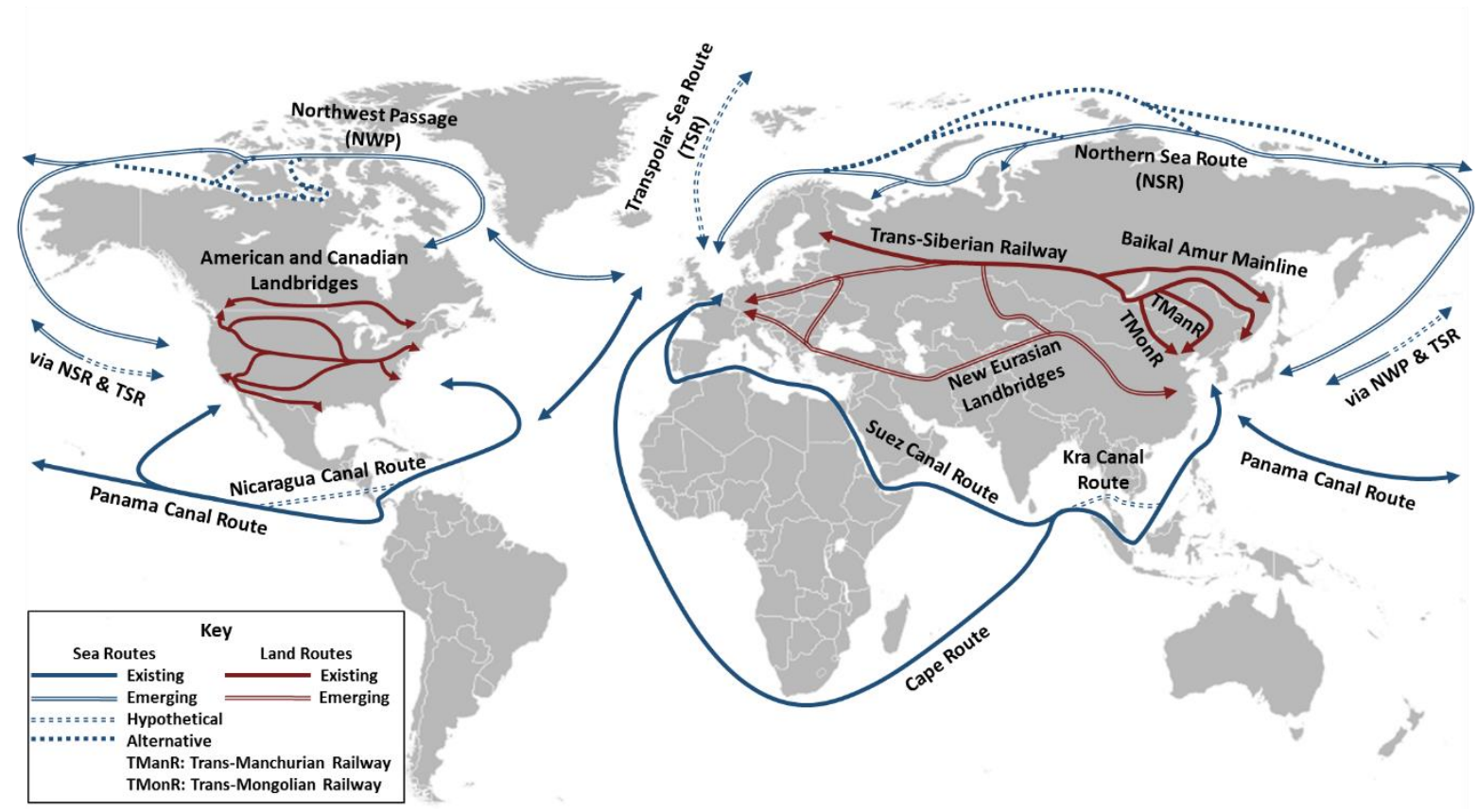

Fig.3. Alternative sea and land routes between Eurasia and North America

(Authors, based on Rodrigue et al., 2017 and MERICS Research, 2017)

\subsubsection{Cost, operational, and navigational variables}

A wide variety of cost, operational, and navigational variables were identified. This is in line with the findings of Lasserre (2014). The differences extend to include not only the unit of cost and emissions measurement but also operational and market factors as well.

To begin with, the assumed sailing season affects the route comparison and the results across the models. This implies that under the current winter navigational and climatic conditions they could serve as seasonal alternatives for a limited period of about five months (summer season) mainly for bulkers and tankers rather than offering regular access to ships on an annual basis. It was identified that a combination of an extended sailing season with low ice-breaking fees increases significantly the competitiveness of the Arctic routes even under high fuel prices (Liu and Kronbak, 2010, Lasserre 2014, 2015, Zhao et al., 2016) and use of larger vessels on the traditional routes (Furuichi and Otsuka, 2015). Further, increased load factors and high average speeds that enable many rotations through the Arctic routes, could improve profitability, especially for liner shipping operations (Wergeland, 1992, Guy, 2006, Lasserre, 2014, 2015).

The capital cost premium is in the order of $20-30 \%$ in most cases and was identified as an important cost factor amongst others (Somanathan et al., 2007, 2009, Liu and Kronbak, 2010). The importance of an extended sailing season is crucial in order to exploit the advantages of operating on shorter routes by utilising ice class vessels, which entail increased capital costs. Besides, operators may seek opportunities to use ice class tonnage in other ice bound regions with easier ice conditions during the winter season (e.g. Baltic Sea, Sea of Okhotsk), depending on the ice class and vessel characteristics.

In most of the cases, crew costs were assumed to be $10 \%$ higher when operating in the Arctic routes whilst in Song and Zhang (2013) they were 28\% higher than in the southern routes. 
According to Somanathan et al. (2007), a well-trained crew is required for Arctic operations whilst additional costs occur when ice navigators or additional crew are included as well.

Furthermore, it is widely accepted that insurance costs are higher for ice-class vessels operating in the Arctic. However, a common denominator is difficult to find since each voyage in Arctic waters is evaluated individually. According to a recent survey by Sarrabezoles et al. (2016), most of the insurers stated that H\&M premiums range between 25 and $50 \%$, others estimated them between 0 and $25 \%$, whereas only one assumed rates between 50 and $75 \%$. As regards the P\&I and cargo insurance premiums, these range from 0 to $25 \%$ most of the times whilst an almost equal number of respondents estimated cargo insurance premiums around $25-50 \%$.

The ice breaking fees assumed in the reviewed papers can be distinguished in two broad categories. Those, which refer to the official NSRA fees, and those, which refer to discounts, offered in particular cases from time to time. This discrepancy stems from the fact that transit fees have been subject to fluctuations related to financial and geostrategic reasons rather than a well-targeted policy during the period 1991-2013 (Gritsenko and Kiiski, 2016). Several studies emphasise the importance of relatively low ice breaking fees in order for the Arctic routes to be viable (Liu and Kronbak, 2010, Zhao et al., 2016, Lasserre, 2014, 2015, Furuichi and Otsuka, 2015). On the other hand, the fact that ice-breaking assistance is not compulsory since 2012 and the recent improvements on navigational rules implemented in 2014 are remedies to the aforementioned obstacles (Gritsenko and Kiiski, 2016).

The average speed used in the models also differs widely. The operating speed depends on the speed realised on ice waters since the speed in open waters will be the same as in classical routes (Faury and Cariou, 2016). This variability in sailing speeds stemming from the uncertainty of ice and weather conditions underline that there are no standards and every case is unique. According to the NSRA data, the average speed recorded between 2011-2014 is around $10 \mathrm{kts}$ (NSRIO, 2018), which is in line with the operating speeds realised in first-year sea ice in the Bay of Bothnia during the ice season. However, it can be easily reduced to 5-6 kts or even to zero depending on the ice and local climatic conditions.

Some of the studies assume increased fuel consumption in their scenarios due to greater engine power required in the icy parts of the route and the additional weight of an ice class vessel. Whilst the use of the shorter Arctic routes imply lower fuel costs, this largely depends on transit times and possible delays due to deviation of a vessel from its predefined navigational route in order to avoid difficult ice conditions. Pruyn (2016) suggest that fuel consumption of an unescorted vessel should be equal to that at design speed regardless of the speed realised on ice. Further research could shed light on the fuel consumption of an ice class vessel in both open water and ice to refine cost simulations with respect to overall fuel costs when comparing ice-infested and traditional routes.

As regards the types of fuel used by ships in the Arctic routes, Lasserre $(2014 ; 2015)$ claimed that IFO 380 fuel may not be appropriate during winter navigation. The picture becomes more blurred if possible taxation on fuel is assumed (Schøyen and Bråthen, 2014), future emissions surcharges (Carriou and Faury, 2015), the use of alternative fuels in the Arctic or the extension of a recent IMO policy that requires vessels to use fuels containing less than $0.1 \%$ sulphur in ECAs such as the North and Baltic seas to the Polar Circle (Lindstad et al., 2016). Moreover, alternative approaches of estimating the environmental impact of maritime operations on different regions my give different results. Lindstad et al (2016) challenged the assessment of $\mathrm{CO}_{2}$ emissions that rely merely on fuel consumption conversion to $\mathrm{CO}_{2}$ amounts when considering the Arctic region. They claimed that, if region-specific Global Warming Potential 
(GWP) factors are applied, then the NSR generates higher $\mathrm{kg}-\mathrm{CO}_{2}$-eq/t than the Suez Canal route even if LNG is used as a fuel.

\subsubsection{Revenue and market factors}

The literature focuses mainly on cost and navigational factors, whilst revenue factors have been overlooked in most cases. Shipowners adjust the operating speed according to the prevailing market conditions and fuel prices amongst others. Thus, a combination of low freight rates and high bunker prices impose speed reductions which favour slow steaming and vice versa (Notteboom and Vernimmen, 2009, Devanney, 2010). The reduction in voyage costs in this case is very small and other factors such as low load factors, high ice breaking fees or potential delays and uncertainty when it comes to liner shipping, favour the classical routes (Liu and Kronbak, 2010, Lasserre, 2014, 2015, Zhang et al., 2016). Further, the relationship between speed, freight rates and fuel prices is not often straightforward if other factors such as cargo value, in-transit inventory and operating costs are included amongst others. The net effect of these factors upon the route choice depends critically on the logistical context of the calculations. Commodity prices and proximity to the markets play an important role too. Differences in the distances explain some of the differences in freight rates and regional commodity prices amongst others (Laulajainen, 2007, Maxwell and Zhu, 2011). Economies of scale and different ship sizes and ice class designs are also important factors, which have not been investigated thoroughly in the literature.

\subsubsection{Maritime transport systems}

Whilst 20 studies assessed the Arctic routes against liner shipping, this system seems to be the most uneconomical and unfeasible to date. Thus, a number of criteria are not satisfied when it comes to carrier's port selection: remote geographical location of Arctic ports, lack of proximity to markets and access to hinterlands, regional bottlenecks and port infrastructure and service (Lirn et al., 2004; Song and Yeo, 2004). Other factors are high uncertainty related to ice and climatic conditions in the Arctic that may result in delays, schedule unreliability and longer transit times (Zohil and Prijon, 1999; Notteboom, 2006).

Some of the reviewed papers partly tackle these issues by incorporating lower load factors to both eastbound and westbound cargoes. Although Arctic routes do not provide for sufficient port calling, they can serve as shorter routes in the long-term, allowing liner operators to either reconfigure their networks or establish separate services through the Arctic. The global geographical focus of liner networks as well as the inherent trend of expansion in secondary markets (Guy, 2003; Baird, 2006) will possibly trigger the interest of operators possessing large capacity to extend their network in the Arctic by establishing seasonal transits in the short-term and forming regular networks in the long-term (Lee and Kim, 2015). Few studies report a network structure in the literature (Xu et al., 2011, Tavasszy et al., 2011, Fan et al., 2012, 2015, Zhao et al., 2016). Further research could shed light on network structure and the feasibility of liner operations from this perspective.

It is evident from this review that bulk and specialised shipping are both overlooked in most of the papers (11 of 33). This neglects the possibility for the Arctic routes to emerge as an alternative option for liquid and dry bulk trades and other cargoes, especially oil, gas condensate, naphtha, LNG and to a lesser extent iron ore and other minerals (Jørgensen-Dahl and Wergeland, 2013; Bambulyak et al., 2015). The potential of bulk and specialised shipping was also reported in recent surveys (Lasserre and Pelletier, 2011; Lee and Kim, 2015; Beveridge et al., 2016; Lasserre et al., 2016). The reefer segment is also largely neglected in 
the literature. However, the NSRA statistics show that there are a considerable number of reefer vessels transporting frozen fish via the NSR (NSRIO, 2018). Thus, economies of short distances offered by the NSR could be materialised concerning locations between North Europe and Northeast Asia as regards reefer vessels.

\subsection{Methodological insights}

The methods and data analysis techniques identified in the literature are discussed in this section to provide insights on how these could be developed in the future. Techniques that could aid modelling in this area are also presented, although other techniques could be appropriate. As a relatively new topic in maritime transport area, Arctic shipping, could be addressed by many methods and techniques used in social sciences.

\subsubsection{Operational research and cost modelling approaches}

Arctic shipping could be a fertile ground for operational research techniques. Examples are the comparative study of Zhao et al. (2016) who bring together the areas of liner network design and Arctic shipping, and Fan et al. $(2012 ; 2015)$ where linear programming and stochastic network models were employed to minimise costs and assess risk uncertainties with respect to future container flows. Most of the papers reviewed in this systematic review consider the assignment of one vessel in single or annual voyages. Operational research methods could increase the parameters of the models by considering several alternative options related to fleet size, route choice, the number of voyages and networks (Fan et al. 2012; 2015). Contemporary operational tactics such as sailing speed adjustments adopted to minimise fuel consumption and/or costs or to maximise profits in both liner and tramp shipping are very relevant (Psaraftis and Kontovas, 2013). Environmental sustainability is also addressed through multi-objective optimisation techniques (Mansouri et al., 2015). Thus, modelling could be informed from all the aforementioned techniques to address operational, economic and environmental aspects. As Zhao et al. (2016) mentioned, the majority of operational research studies in shipping rely on the established routes and networks and do not consider new routes and their impact on maritime operations.

Given that scenario-based transport cost models reported in the literature are likely to remain the prevalent technique, they could be developed further to include more assumptions considering not only operational and cost factors but also environmental factors. Of the 33 studies reviewed, only eight report the estimation of emissions based on various formulas. Studies within climate science evaluating future accessibility in the Arctic could also aid the modelling approaches with respect to navigation season, sea ice conditions and transit times so as to better quantify these factors. Global climate models projecting ice and weather conditions under different emissions scenarios could be used as inputs to simulations (Schröder et al., 2017). On the other hand, more diversity is needed in terms of scenarios and assumptions so as to provide fruitful insights and counter-arguments. Arctic maritime operations require cost analysis methods that can deal with the structure and complexity of the issues being involved.

\subsubsection{Empirical case studies}

Many authors mention the discrepancies in hypotheses and assumptions made in the literature regarding operational and cost variables as well as market conditions (Lasserre, 2014; Cariou and Faury, 2015). This is a result of the infancy of Arctic maritime operations, which in turn leads to the lack of relevant data and statistics. Empirical case studies with a focus on the examination of the Arctic routes and interviews with key stakeholders can complement the data reported in databases and other publicly available sources to further refine any modelling 
approach where there is no, insufficient, or inaccurate statistical data. The identified case studies report empirical data obtained through interviews and records from real voyages occurred in the NSR (Raza and Schøyen, 2014, Zhao and Hu, 2016). This type of research could help increase the understanding of Arctic maritime operations. As Wacker (1998) pointed out, empirical research offers verification of model-based research amongst others. In particular, case studies provide a deeper understanding of the usually complex operational processes in the real world.

\subsubsection{Econometric modelling, regression, panel data analysis and other techniques}

Structural econometric modelling, regression and panel data analysis have been widely used in maritime research (Glen, 2006; Heaver, 2012; Xu and Yip, 2012). All these techniques could be employed in order to develop models that test various explanatory variables with respect to the determination of costs, profits and emissions when utilising maritime routes. For instance, Lu et al (2014) use explanatory variables such as freight rates, distance, time, transit fees, fuel consumption and vessel size in order to investigate the cost determinants on both the NSR and the Suez Canal route. Pruyn (2016) uses a sophisticated structural model including macroeconomic data from sixteen countries, vessel sizes, fleet age, freight rates and transport costs to explore the feasibility of the NSR. Other techniques such as discrete choice and MCDM models could aid model-based research by investigating stakeholders' perspectives regarding influential decision-making factors and the potential of Arctic shipping amongst others (Moon et al., 2015, Shyu and Ding, 2016, Benedyk and Peeta, 2016, Wang et al., 2018).

\section{Conclusion}

The literature on comparative studies between Arctic and traditional routes has grown considerably during the last ten years. Whilst there have been attempts to identify, survey (Lasserre, 2014; 2015) and review the extant literature (Meng et al., 2016), to date there have been no systematic reviews. This study therefore contributes to Arctic shipping research by providing the first such systematic review of the literature between 1980 and 2017.

In doing so, both economic issues and environmental assessment of the Arctic routes were taken into account. Further, the methodological characteristics of the reviewed studies were identified and analysed. In addition, important decision-making factors were pointed out and discussed, such as cost (capital cost premiums, ice breaking fees, fuel costs, operating costs, in-transit inventory costs), operational (speed, fuel types), navigational (alternative routes, sea ice conditions) and revenue and market factors (freight rates, commodity prices, cargo value). This review serves as the starting point of developing a conceptual framework of route choice decision-making factors within the context of Arctic shipping which could be used in future research.

The results of this review suggest that Arctic routes are considered more competitive than their traditional rivals in 13 of the 31 papers that evaluated their economic potential. On the other hand, they were found to be less competitive in six papers whereas seven reported mixed results. Five papers project that they will become competitive in the long-term. Only eight studies assessed emissions comparing the Arctic with traditional routes, with seven out of eight studies concluding that Arctic shipping routes are more energy efficient than their traditional rivals. The competitiveness of these routes decreases as we move towards year-round operations. This means that under the current winter navigational and climatic conditions they could serve mainly as seasonal alternatives for a limited period of about five months rather than offering regular access to ships on an annual basis. Consequently, Arctic routes appear to be 
more suitable for bulk rather than liner shipping in the short to medium-term. The findings serve as evidence to inform transport practitioners who operate or willing to operate in the Arctic regarding cost, revenue, operational and navigational factors. Moreover, they provide an initial understanding of what factors promote or hinder the cost-competitiveness and/or profitability of the Arctic routes, why and how.

This review identified several issues that need to be addressed in future research. These relate to both research and methodological aspects of the reviewed papers. Further research is required in terms of the NWP, the TSR and variations of the NSR. Attention should be paid to revenue attributes, commodity and fuel prices, and how these factors along with Arctic sea ice conditions determine the competitiveness of the Arctic routes. More model-based research with robust sensitivity analyses is needed in order to overcome discrepancies in the assumptions regarding cost and operational variables. With respect to the navigational factors, future research could take into account studies related to climate change models and variations in the Arctic sea ice. Ice conditions as well as other physical constraints (e.g. regional bottlenecks) are critical factors that affect the operating speed or the size of the vessels used in Arctic waters, which in turn affect the revenue, transit time, operating and voyage costs.

The literature focuses mainly on liner shipping and to a lesser extent on bulk shipping. However, bulk (Liquid, Dry) and specialised shipping (LNG, Reefer) will mostly benefit from Arctic routes in the short to medium-term. For liner shipping, more emphasis could be given to network structure/configuration and/or reconfiguration of the existing networks as part of scenario-based simulations. Analytical mathematical methods were found to be prevalent in the literature with empirical statistical and case study research being used in a lesser extent. With regards to data analysis techniques found during the review, the literature shows a particular preference for scenario-based transport cost models. When it comes to the methodological aspects, this study suggests that as a relatively new topic in maritime transport area, Arctic shipping could be addressed by many methodologies and techniques used in social sciences, namely, operational research, case studies, econometric modelling, regression and panel data analysis as well as discrete choice and MCDM techniques amongst others. Finally, this review limited its scope to studies reporting on cost/emissions assessment of Arctic routes. However, the Arctic shipping literature spans several research areas and topics. A broader review of the literature could include conceptual and descriptive studies, surveys and studies focusing on factors other than costs/profits (e.g. time/distance effects or ice class ship evaluation) or on the overall environmental impact of future shipping traffic volumes in the Arctic.

\section{Acknowledgements}

This work was supported by the Economic and Social Research Council [ESRC Wales Doctoral Training Partnership].

\section{References}

Aksenov, Y., Popova, E.E., Yool, A., Nurser, A.J.G., Williams, T.D., Bertino, L., Bergh, J., 2017. On the future navigability of Arctic sea routes: High-resolution projections of the Arctic Ocean and sea ice. Marine Policy 75, 300-317. 
Baird, A., 2006. Optimising the container transhipment hub location in northern Europe. Journal of Transport Geography 14 (3), 195-214.

Bambulyak, A., Frantzen, B., Rautio, R., 2015. Oil transport from the Russian part of the Barents Region: 2015 status report. The Norwegian Barents Secretariat-Akvaplanniva AS, Norway.

Benedyk, I.V., Peeta, S., 2016. A binary probit model to analyze freight transportation decision-maker perspectives for container shipping on the Northern Sea Route. Maritime Economics and Logistics (Online), 1-17.

Beveridge, L., Fournier, M., Lasserre, F. Huang, L., Têtu, P-L., 2016. Interest of Asian shipping companies in navigating the Arctic. Polar Science 10 (3), 404-414.

Carriou, P., Faury, O., 2015. Relevance of the Northern Sea Route (NSR) for bulk shipping. Transportation Research Part A 78, 337-346.

Chang, K.Y., He, S.S., Chou, C.C., Kao, S.L., Chiou, A.S., 2015. Route planning and cost analysis for travelling through the Arctic Northeast Passage using public 3D GIS.

International Journal of Geographical Information Science 29 (8), 1375-1393.

Chou, C-C., Shyu, W-H., Chen, C-Y., Hsiao, H-H., 2015. The impact on the operation costs of bulk ship after the opening of the Arctic route. Advanced Science Letters 21 (11), 3594-3596.

Chou, M-T., Chou, T-Y., Hsu, Y-R., Lu, C-P., 2017. Fuel consumption ratio analysis for transiting from various ports and harbours in Asia through the Northern Sea Route. The Journal of Navigation 70 (3), 859-869.

Corbett, J.J., Wang, H., Winebrake, J.J., 2009. The effectiveness and costs of speed reductions on emissions from international shipping. Transportation Research Part D: Transport and Environment 14 (8), 593-598.

David, R.J., Han, S.K. A systematic assessment of the empirical support for transaction cost economics. Strategic Management Journal 25 (1), 39-58.

De Marucci, S., 2012. The expansion of the Panama Canal and its impact on global $\mathrm{CO}_{2}$ emissions from ships. Maritime Policy \& Management 39 (6), 603-620.

Devanney, J., 2010. The Impact of Bunker Price on VLCC Spot Rates. Proceedings of the 3rd International Symposium on Ship operations, Management and Economics, SNAME Greek Section, Athens, 7-8 October.

Ducruet, C., 2013. Network diversity and maritime flows. Journal of Transport Geography 30, 77-88.

Eguíluz, V.M., Fernández-Gracia, J., Irigoien, X., Duarte, C.M., 2016. A quantitative assessment of Arctic shipping in 2010-2014. Scientific Reports (6), 1-6.

Fan, L., Wilson, W.W., Dahl, B., 2012. Impacts of new routes and ports on spatial competition for containerized imports into the United States. Maritime Policy \& Management 35 (9), 479501.

Fan, L., Wilson, W.W., Dahl, B., 2015. Risk analysis in port competition for containerized imports. European Journal of Operational Research 245 (3), 743-753. 
Faury, O., Carriou, P., 2016. The Northern Sea Route competitiveness for oil tankers. Transportation Research Part A: Policy and Practice 94, 461-469.

Furuichi, M., Otsuka, N., 2015. Proposing a common platform of shipping cost analysis of the Northern Sea Route and the Suez Canal Route. Maritime Economics and Logistics 17 (1), 931.

Glen, D.R., 2006. The modelling of dry bulk and tanker markets: a survey. Maritime Policy \& Management 33 (5), 431-445.

Gritsenko, D., Kiiski, T., 2016. A review of Russian ice-breaking tariff policy on the northern sea route 1991-2014. Polar Record 52 (2), 144-158.

Guy, E., 2003. Shipping line networks and the integration of South America trades. Maritime Policy \& Management 30 (3), 231-242.

Guy, E., 2006. Evaluating the viability of commercial shipping in the Northwest Passage. Journal of Ocean Technology 1 (1), 9-15.

Heaver, T., 2012. The evolution of maritime economics. In: W. Talley (Ed.), The blackwell companion to maritime economics, Blackwell Publishing Ltd, Sussex, pp.16-33.

Jørgensen-Dahl, A., Wergeland, T., 2013. Shipping, resources, economic trends and alternative means of transport. In: Østreng, W., Eger, K.M., Fløistad, B., Jørgensen-Dahl, A., Lothe, L., Mejlaender-Larsen, M., Wergeland, T. (Eds.), Shipping in Arctic Waters: A comparison of the Northeast, Northwest and Trans Polar Passages, Springer, pp. 83-145.

Khon, V.C., Mokhov, I.I., 2010. Arctic climate changes and possible conditions of Arctic navigation in the 21st century. Izvestiya, Atmospheric and Oceanic Physics 46 (1), 19-25.

Khon, V.C., Mokhov, I.I., Latif, M., Semenov, V.A., Park, W., 2010. Perspectives of Northern Sea Route and Northwest Passage in the twenty-first century. Climatic Change 100 (3-4), 757 768.

Khon, V.C., Mokhov, I.I., Semenov, V.A., 2017. Transit navigation through Northern Sea Route from satellite data and CMIP5 simulations. Environmental Research Letters 12 (20).

Knowles, R.D., 2006. Transport shaping space: differential collapse in time-space. Journal of Transport Geography 14 (6), 407-425.

Kondo, K., Takamasa, T., 1999. The economic potential of a cassette-type-reactor-installed nuclear ice-breaking container ship. Journal of Nuclear Science and Technology. 36 (12), 11991208.

Laliberté, F., Howell, S.E.L., Kushner, P.J., 2016. Regional variability of a projected sea icefree Arctic during the summer months. Geophysical Research Letters 43 (1), 256-263.

Lam, J.S.L., 2010. An integrated approach for port selection, ship scheduling and financial analysis. NETNOMICS: Economic Research and Electronic Networking 11 (1), 33-46.

Lasserre, F., 2014. Case studies of shipping along Arctic routes. Analysis and profitability perspectives for the container sector. Transportation Research Part A 66, 144-161.

Lasserre, F., 2015. Simulations of shipping along Arctic routes: comparison, analysis and economic perspectives. Polar Record 51 (3), 239-259. 
Lasserre, F., Pelletier, S. 2011. Polar super seaways? Maritime transport in the Arctic: an analysis of shipowners' intentions. Journal of Transport Geography 19 (6), 14651473.

Lasserre, F., Beveridge, L., Fournier, M., Têtu, P-L., Huang, L., 2016. Polar super seaways? Maritime transport in the Arctic: An analysis of shipowners' intentions II. Journal of Transport Geography 57, 105-114.

Laulajainen, R., 2007. Dry bulk shipping inefficiency, the wide perspective. Journal of Transport Geography 15 (3), 217-224.

Lee, T., Kim, H.J., 2015. Barriers of voyaging on the Northern Sea Route: A perspective from shipping companies. Marine Policy 62, 264-270.

Lindstad, H., Jullumstrø, E., Sandaas, I., 2013. Reductions in cost and greenhouse gas emissions with new bulk ship designs enabled by the Panama Canal expansion. Energy Policy 59, 341-349.

Lindstad, H., Bright, R.M., Strømman, A.H., 2016. Economic savings linked to future Arctic shipping trade are at odds with climate change mitigation. Transport Policy 45, pp. 24-30.

Lirn, T-C, Thanopoulou, H.A., Beynon, M.J., Beresford, A.K.C., 2004. An Application of AHP on Transhipment Port Selection: A Global Perspective. Maritime Economics \& Logistics 6 (1), 70-91.

Liu, M., Kronbak, J., 2010. The potential economic viability of using the Northern Sea Route (NSR) as an alternative route between Asia and Europe. Journal of Transport Geography 18 (3), 434-444.

Liu, X., Ma, L., Wang, J., Wang, Y., Wang, L., 2017. Navigable windows of the Northwest Passage. Polar Science 13, 91-99.

Lu, D., Park, G-K., Choi, K., Oh, K., 2014. An Economic Analysis of Container Shipping through Canadian Northwest Passage. International Journal of e-Navigation and Maritime Economy 1, 60-72.

Mansouri, S.A., Lee, H., Aluko, O., 2015. Multi-objective decision support to enhance environmental sustainability in maritime shipping: A review and future directions. Transportation Research Part E: Logistics and Transportation Review 78, 3-18.

Martinez, C., Steven, A.B., Dresner, M., 2016. East Coast vs. West Coast: The impact of the Panama Canal's expansion on the routing of Asian imports into the United States. Transportation Research Part E: Logistics and Transportation Review 91, 274289.

Maxwell, D., Zhu, Z., 2011. Natural gas prices, LNG transport costs and the dynamics of LNG imports. Energy Economics 33 (6), 217-226.

Melia, N., Haines, K., Hawkins, E., 2016. Sea ice decline and 21st century trans-Arctic shipping routes. Geophysical Research Letters 43 (18), 9720-9728.

Meng, Q., Zhang, Y., Xu, M., 2016. Viability of transarctic shipping routes: a literature review from the navigational and commercial perspectives. Maritime Policy \& Management 44 (1), 1-26.

Meredith, J.R., Raturi, A., Amoako-Gyampah, K., Kapplan, B., 1989. Alternative research paradigms in operations. Journal Of Operations Management 8 (4), 297-326. 
MERICS Research, 2017. The Silk Road initiative. < https://www.merics.org/en/chinamapping/silk-road-initiative> (accessed 27.03.2018).

Moon, D-S., Kim, D-J., Lee, E-K., 2015. A study on competitiveness of sea transport by comparing international transport routes between Korea and EU 31 (1), 1-20.

Notteboom, T.E., 2006. The time factor in liner shipping services. Maritime Economics \& Logistics 8 (1), 19-39.

Notteboom, T.E., Vernimmen, B., 2009. The effect of high fuel costs on liner service configuration in container shipping. Journal of Transport Geography 17 (5), 325-337.

Notteboom, T.E., 2012. Towards a new intermediate hub region in container shipping? Relay and interlining via the Cape route vs. the Suez route. Journal of Transport Geography 22, 164178.

NSRIO, 2018. Transit statistics. 〈http://www.arctic-lio.com/nsr_transits $>$ (accessed 20.03.2018).

Pizzolato, L., Howell, S. E. L., Dawson, J., Laliberté, F., Copland, L., 2016. The influence of declining sea ice on shipping activity in the Canadian Arctic. Geophysical Research Letters 43 (23), pp. 12,146-12,154.

Pruyn, J.F.J., 2016. Will the Northern Sea Route ever be a viable alternative? Maritime Policy \& Management 43 (6), 1-15.

Psaraftis, H.N., Kontovas, C.A., 2013. Speed models for energy-efficient maritime transportation: A taxonomy and survey. Transportation Research Part C 26, 331-351.

Raza, Z., Schøyen, H., 2014. The commercial potential for LNG shipping between Europe And Asia via the northern sea route. Journal of Maritime Research XI (II), 6779.

Rodrigue, J-P., Ashar, A., 2016. Transshipment hubs in the New Panamax Era: The role of the Caribbean. Journal of Transport Geography 51, 270-279.

Rodrigue, J-P., Comtois, C., Slack, B., 2017. The geography of transport systems, 4th ed. Routledge, London.

Sachan, A., Datta, S., 2005. Review of supply chain management and logistics research. International Journal of Physical Distribution \& Logistics Management 35 (9), 664-705.

Sarrabezoles, A., Lasserre, F., Hagouagn'rin, Z., 2016. Arctic shipping insurance: towards a harmonisation of practices and costs? Polar Record 52 (265), 393-398.

Schøyen, H., Bråthen, S., 2011. The Northern Sea Route versus the Suez Canal: cases from bulk shipping. Journal of Transport Geography 19 (4), 977-983.

Schröder, C., Reimer, N., Jochmann, P., 2017. Environmental impact of exhaust emissions by Arctic Shipping. Ambio 46 (3), 400-409.

Smith, L.C., Stephenson, S.R., 2013. New Trans-Arctic shipping routes navigable by midcentury. Proceedings of the National Academy of Sciences of the United States of America 110 (13), 4871-4872.

Somanathan, S., Flynn, P.C., Szymanski, J.F., 2007. Feasibility of a sea route through the Canadian Arctic. Maritime Economics \& Logistics 9 (4), 324-334. 
Somanathan, S., Flynn, P., Szymanski, J., 2009. The Northwest Passage: A simulation. Transportation Research Part A 43 (2), 127-135.

Song, D-W., Yeo, K-T., 2004. A competitive analysis of Chinese container ports using the analytic hierarchy process. Maritime Economics \& Logistics 6 (1), 34-52.

Song, Y., Zhang, A., 2013. The economy analysis of sailing in the Arctic Northeast Passage. Applied Mechanics and Materials 409-410, 1253-1257.

Stephenson, S.R., Smith, L.C., Brigham, L.W., Agnew, J.A., 2013. Projected 21st-century changes to Arctic marine access. Climatic Change 118 (3-4), 885-899.

Stephenson, S.R., Brigham, L.W., Smith, L.C., 2014. Marine accessibility along Russia's Northern Sea Route. Polar Geography 37 (2), 111-133.

Stephenson, S. R., Smith, L.C., 2015. Influence of climate model variability on projected Arctic shipping futures. Earth's Future 3 (11), 331-343.

Stopford, M., 2009. Maritime Economics, 3rd ed. Routledge, United Kingdom.

Shyu, W-H, Ding, J-F., 2016. Key factors influencing the building of Arctic shipping routes. The Journal of Navigation 69 (6), 1261-1277.

Tavasszy, L., Minderhoud, M., Perrin, J-F., Notteboom, T., 2011. A strategic network choice model for global container flows: specification, estimation and application. Journal of Transport Geography 19 (6), 1163-1172.

Tranfield, D., Denyer, D., Smart, P., 2003. Towards a methodology for developing evidenceinformed management knowledge by means of systematic review. British Journal of Management 14 (3), 207-222.

Verny, J., Grigentin, C., 2009. Container shipping on the Northern Sea Route. International Journal of Production Economics 122 (1), 107-117.

Wacker, J.G., 1998. A definition of theory: research guidelines for different theory building research methods in operations management. Journal of Operations Management 16 (4), 361385 .

Wang, N., Tan, B., Wu, N., Zhao, W-J., 2016. Comments on "Case studies of shipping along Arctic routes. Analysis and profitability perspectives for the container sector". Transportation Research Part A: Policy and Practice 94, 699-702.

Wang, H, Zhang, Y., Meng, Q. 2018. How will the opening of the Northern Sea Route influence the Suez Canal Route? An empirical analysis with discrete choice models. Transportation Research Part A: Policy and Practice 107, 75-89.

Wergeland, T., 1992. The northern sea route - rosy prospects for commercial shipping? International Challenges 12 (1), 43-57.

Woo, S. H., Pettit, S.J., Kwak, D.W., Beresford, A.K.C., 2011. Seaport research: A structured literature review on methodological issues since the 1980's. Transportation Research Part A 45 (7), 667-685.

Xu, H., Yin, Z., Jia, D., Jin, F., Ouyang, H., 2011, The potential seasonal alternative of AsiaEurope container service via Northern sea route under the Arctic sea ice retreat. Maritime Policy \& Management 38 (5), 541-560. 
Xu, J.J., Yip, T.L., 2012. Ship investment in a standstill? An analysis of shipbuilding activities and policies. Applied Econometrics Letters 19 (3), 269-275.

Yip, T.Z., Wong, M.C., 2016. The Nicaragua Canal: scenarios of its future roles. Journal of Transport Geography 43, 1-13.

Zeng, Q., Wang, G.W.Y., Qu, C., Li, K.X., 2017. Impact of the Carat Canal on the evolution of hub ports under China's Belt and Road initiative. Transportation Research Part E: Logistics and Transportation Review (In press).

Zhang, Y., Meng, Q., Ng, S.H., 2016. Shipping efficiency comparison between northern sea route and the conventional Asia-Europe shipping route via Suez Canal. Journal of Transport Geography 57, 241-249.

Zhang, Y., Meng, Q., Zhang, L., 2016. Is the Northern Sea Route attractive to shipping companies? Some insights from recent ship traffic data. Marine Policy (73), 53-60.

Zhao, H., Hu, H., 2016. Study on economic evaluation of the northern sea route: taking the voyage of Yong Sheng as an example. Transportation Research Board 2549, 78-85.

Zhao, H., Hua, H., Lin, Y., 2016. Study on China-EU container shipping network in the context of Northern Sea Route. Journal of Transport Geography 53, 50-60.

Zohil, J., Prijon, M., 1999. The MED rule: the interdependence of container throughput and transhipment volumes in the Mediterranean ports. Maritime Policy \& Management 26 (2), 175193. 
Appendix. Factors affecting ship revenues and $\operatorname{costs}^{1}$

\begin{tabular}{|c|c|c|c|c|c|c|}
\hline $\begin{array}{l}\text { Author(s) } \\
\text { and year }\end{array}$ & Crew premium & Insurance premium & Transit fees & $\begin{array}{l}\text { Fuel consumption } \\
\text { rate }\end{array}$ & Speed & $\begin{array}{l}\text { Capital Cost } \\
\text { Premium }\end{array}$ \\
\hline $\begin{array}{l}\text { Wergeland } \\
\text { (1991) }\end{array}$ & $+17.3 \%$ & $\begin{array}{l}+25 \% \text {, Cargo insurance: } \\
0.2 \% \text { per voyage }\end{array}$ & $\begin{array}{l}\text { Official NSRA } \\
\text { fees }\end{array}$ & N.A. & $\begin{array}{l}\text { NSR: } 12 \text { kts, Suez \& } \\
\text { Panama Canal: } 13.5 \text { kts }\end{array}$ & $+35 \%$ \\
\hline $\begin{array}{l}\text { Kondo and Takamasa } \\
(1999)^{2}\end{array}$ & $+147 \%$ & $\begin{array}{l}+183.7 \% \\
+936,000 \text { US\$ nuclear } \\
\text { energy insurance/year }\end{array}$ & N.A. & N.A. & $\begin{array}{l}\text { NSR: } 20 \text { kts } \\
\text { Suez Canal: } 25,30 \\
\& 34.2 \text { kts }\end{array}$ & $+134.4 \%$ \\
\hline Guy (2006) & $\begin{array}{l}+15-200 \% \text { premium incl. } \\
\text { in Trip Charter costs }\end{array}$ & $\begin{array}{l}+15-200 \% \text { premium incl. } \\
\text { in Trip Charter costs }\end{array}$ & Hypothetical & N.A. & $\begin{array}{l}\text { Optimistic Scenario: } \\
22 \text { kts, Pessimistic } \\
\text { Scenario: } 6 \text { kts on ice }\end{array}$ & $\begin{array}{l}+15-200 \% \text { premium incl. } \\
\text { in Trip Charter costs }\end{array}$ \\
\hline $\begin{array}{l}\text { Somanathan et al. } \\
(2007)\end{array}$ & +565 US\$/day & $+50-51.4 \%$ & No fees & $\begin{array}{l}\text { Same SFOC but higher } \\
\text { engine power }\end{array}$ & $\begin{array}{l}\text { NWP \& Panama Canal: } \\
11 \& 20 \text { kts }\end{array}$ & $+30 \%$ \\
\hline $\begin{array}{l}\text { Somanathan et al. } \\
\text { (2009) }\end{array}$ & $+21.2 \%$ & $+50 \%$ & No fees & $\begin{array}{l}\text { New York-Yokohama: } \\
+50 \%, \text { St Johns, } \\
\text { Newfoundland- } \\
\text { Yokohama: }+58 \%\end{array}$ & $\begin{array}{l}\text { New York/ St. Johns - } \\
\text { Yokohama: NWP: } 18.4 \\
\text { kts (September) } \\
\text { 18.2 kts (February), } \\
\text { Panama Canal: } 20 \text { kts }\end{array}$ & $+30 \%$ \\
\hline $\begin{array}{l}\text { Verny and Grigentin } \\
(2009)\end{array}$ & N.A. & N.A. & $\begin{array}{l}\text { Official NSRA } \\
\text { fees }\end{array}$ & N.A. & $\begin{array}{l}\text { NSR: } 17 \mathrm{kts} \text { on ice \& } \\
24 \mathrm{kts} \text { on open water, } \\
\text { SCR: } 24 \mathrm{kts}\end{array}$ & N.A. \\
\hline $\begin{array}{l}\text { Liu and Kronbak } \\
\text { (2010) }\end{array}$ & $+10 \%$ & $+62.5 \%$ & $\begin{array}{l}\text { Official NSRA } \\
\text { fees \& three } \\
\text { variant scenarios }\end{array}$ & $\begin{array}{l}\text { SCR: } 0.3 \mathrm{t} / \mathrm{nm} \\
\text { NSR (on ice): } 0.5 \mathrm{t} / \mathrm{nm} \\
=\text { nautical mile }\end{array}$ & $\begin{array}{l}\text { NSR: } 10 \mathrm{kts} \text { on ice } \\
\text { water \& } 18 \mathrm{kts} \text { on open } \\
\text { water, SCR: } 18 \mathrm{kts}\end{array}$ & $+20 \%$ \\
\hline $\begin{array}{l}\text { Schøyen and Bråthen } \\
\text { (2011) }\end{array}$ & $\begin{array}{l}+20 \% \text { premium incl. } \\
\text { in Operating Costs }\end{array}$ & $\begin{array}{l}+125,000 \text { US\$/trip for } \\
\text { both H\&M and P\&I }\end{array}$ & $\begin{array}{l}\text { Official NSRA } \\
\text { fees }\end{array}$ & $\begin{array}{l}\text { Porsgrunn-Shekou } \\
\text { (NSR): } 49 \% \text { and } 78 \% \\
\text { lower than SCR and } \\
\text { Cape respectively, } \\
\text { Narvik - Qingdao } \\
\text { (NSR): } 84 \% \text { lower than } \\
\text { NSR }\end{array}$ & $\begin{array}{l}\text { Porsgrunn-Shekou: } \\
\text { NSR: } 11.5 \text { \& } 8.7 \text { kts, } \\
\text { SCR \& Cape: } 14.4 \text { kts } \\
\text { Narvik-Qingdao: NSR: } \\
\text { 8.3 kts, SCR: } 14.4 \text { kts }\end{array}$ & $+20 \%$ \\
\hline Xu et al. (2011) & N.A. & N.A. & No fees & N.A. & N.A. & No premium \\
\hline Fan et al. (2012) & N.A. & N.A. & N.A. & N.A. & $\begin{array}{l}\text { 3-4 kts on ice water } \\
\text { (around } 1000 \text { n.m.) }\end{array}$ & N.A. \\
\hline $\begin{array}{l}\text { Song and Zhang } \\
\text { (2013) }\end{array}$ & $+28 \%$ & $+5 \%$ & N.A. & $+7 \%$ & $\begin{array}{l}\text { NSR: Based on AIRSS } \\
\text { rules, SCR: } 14.5 \mathrm{kts}\end{array}$ & $+5 \%$ \\
\hline
\end{tabular}

${ }^{1}$ Studies in which these factors could not be discerned are not included in the Appendix.

2 The factors reported in this table refer to the benchmark scenario of a 6,000 TEU diesel ship (20-year life \& speed = 30 kts) compared to a cassette-type MRX ice-breaking 1,400 TEU ship (40-year life) 


\begin{tabular}{|c|c|c|c|c|c|c|}
\hline $\begin{array}{l}\text { Author(s) } \\
\text { and year }\end{array}$ & Crew premium & $\begin{array}{l}\text { Insurance } \\
\text { premium }\end{array}$ & Transit fees & $\begin{array}{l}\text { Fuel consumption } \\
\text { rate }\end{array}$ & Speed & $\begin{array}{l}\text { Capital Cost } \\
\text { Premium } \\
\end{array}$ \\
\hline $\begin{array}{l}\text { Lasserre } \\
(2014)\end{array}$ & $+10 \%$ & $\begin{array}{l}\text { NSR: }+50 \% \\
\text { NWP: }+65 \%\end{array}$ & $\begin{array}{l}\text { NSR: } 7.44 \text { US } \$ / t \\
\text { NWP: No fees }\end{array}$ & $\begin{array}{l}\text { Rotterdam-Shanghai: } \\
\text { NSR and NWP: } 21 \% \\
\text { lower than SCR } \\
\text { Rotterdam- } \\
\text { Yokohama: } \\
\text { NSR and NWP: } 24-25 \% \\
\text { lower than SCR }\end{array}$ & $\begin{array}{l}\text { Rotterdam-Shanghai: } \\
\text { NSR: } 17.71 \text { kts, SCR: } \\
20 \text { kts, NWP: } 16.94 \text { kts } \\
\text { Rotterdam } \\
\text { Yokohama: } \\
\text { NSR: } 16.95 \text { kts, SCR: } \\
\text { 20kts, NWP: } 16.6 \mathrm{kts}\end{array}$ & $+25 \%$ \\
\hline $\begin{array}{l}\text { Raza and Schøyen } \\
\text { (2014) }\end{array}$ & N.A. & $\begin{array}{l}\text { H\&M premium: } \\
+281,250 \text { US } \$ / \text { round } \\
\text { voyage, Increased } \\
\text { Values: }+20,250 \\
\text { US } \$ / \text { round voyage }\end{array}$ & $\begin{array}{l}\text { NSR: } 6.8 \text { US\$/t laden, } 3 \\
\text { US\$/t ballast; SCR: } \\
\text { 158,294US\$/round } \\
\text { voyage (piracy } \\
\text { premium) }\end{array}$ & N.A. & $\begin{array}{l}\text { NSR: } 12 \text { kts on ice } \& \\
19.5 \text { kts on open water } \\
\text { SCR: } 19.5 \mathrm{kts}\end{array}$ & No premium \\
\hline Lu et al. (2014) & N.A. & N.A. & $5 \mathrm{US} \$ / \mathrm{t}$ & N.A. & NWP: 10 kts on ice & N.A. \\
\hline $\begin{array}{l}\text { Lasserre } \\
(\mathbf{2 0 1 5})\end{array}$ & $\begin{array}{l}+10 \% \text { in six-month } \\
\text { scenario } \\
+15 \% \text { in year-round } \\
\text { scenario }\end{array}$ & $\begin{array}{l}\text { Summer season: } \\
\text { NSR: +50\% NWP: } \\
+65 \% \\
\text { Year-round: } \\
\text { NSR: +65\% NWP: } \\
+80 \%\end{array}$ & $\begin{array}{l}\text { NSR: } \\
\text { 1. Official Tariffs } \\
\text { 2. Variant Scenario: } 8.2 \\
\text { US\$/t } \\
\text { NWP: No fees }\end{array}$ & $\begin{array}{l}\text { Summer Season: } \\
\text { Rotterdam-Shanghai: } \\
\text { NSR and NWP: } 21 \% \\
\text { lower than SCR } \\
\text { respectively; } \\
\text { Rotterdam-Yokohama: } \\
\text { NSR: } 25 \% \text { and NWP: } \\
\text { 24\% lower than SCR } \\
\text { Year-round Scenario }\end{array}$ & $\begin{array}{l}\text { Summer Season: } \\
\text { Rotterdam-Shanghai: } \\
\text { NSR: } 17.7 \text { kts, SCR: } 20 \\
\text { kts, } \\
\text { NWP: } 16.9 \text { kts } \\
\text { Rotterdam-Yokohama: } \\
\text { NSR: } 16.9 \text { kts, SCR: } \\
\text { 20kts, NWP: } 16.6 \text { kts } \\
\text { Year-round Scenario }\end{array}$ & $\begin{array}{l}\text { Summer Season: } \\
\text { NSR \& NWP: +20\% } \\
\text { (1AS Ice-class) } \\
\text { Year-round Scenario: } \\
\text { NWP: +30\% (PC4 Ice- } \\
\text { class), NSR: +20\% } \\
\text { (1AS Ice-class) }\end{array}$ \\
\hline $\begin{array}{l}\text { Furuichi and Otsuka } \\
\text { (2015) }\end{array}$ & N.A. & $\begin{array}{l}10 \text { US\$/GT/year } \\
\text { Piracy Premium (SCR): } \\
40 \text { US } \$ / T E U\end{array}$ & $5 \mathrm{US} \$ / \mathrm{GT}$ & $\begin{array}{l}+10 \% \text { SFOC for an ice- } \\
\text { class vessel (additional } \\
\text { weight) }\end{array}$ & $\begin{array}{l}\text { NSR: } 13-14 \text { on ice, } \\
20 \text { kts on open water }\end{array}$ & $+10 \%$ \\
\hline $\begin{array}{l}\text { Chou et al. } \\
\text { (2015) }\end{array}$ & N.A. & N.A. & N.A. & N.A. & $\begin{array}{l}12 \mathrm{kts} \text { in all routes and } \\
\text { cases }\end{array}$ & N.A. \\
\hline $\begin{array}{l}\text { Chang et al. } \\
(2015)\end{array}$ & N.A. & N.A. & Official NSRA fees? & $\begin{array}{l}\text { NSR } 26 \% \text { lower than } \\
\text { SCR on average }\end{array}$ & $\begin{array}{l}\text { NSR: } 14 \text { kts on open } \\
\text { water, } 6-12 \text { kts on ice }\end{array}$ & N.A. \\
\hline $\begin{array}{l}\text { Cariou and Faury } \\
\text { (2015) }\end{array}$ & $\begin{array}{l}+9.5 \% \text { premium incl. in } \\
\text { Operating Costs }\end{array}$ & $+62.5 \%$ & Official NSRA fees & $+5 \%$ & $\begin{array}{l}\text { NSR: } 6.4-12.8 \mathrm{kts} \\
\text { SCR: } 8-16 \mathrm{kts}\end{array}$ & N.A. \\
\hline $\begin{array}{l}\text { Lindstad et al. } \\
\text { (2016) }\end{array}$ & N.A. & N.A. & N.A. & N.A. & NSR \& SCR: 10-11 kts & N.A. \\
\hline
\end{tabular}

3 NWP: 26\% higher (winter) and 19\% lower (summer) than SCR; NSR: 32\% (winter) and 25\% (summer) both lower than SCR

${ }^{4}$ Rotterdam-Yokohama: NWP: 11.7 kts (winter), NSR: 11.2 kts (winter); summer speeds at both routes are the same as in summer season scenarios 


\begin{tabular}{|c|c|c|c|c|c|c|}
\hline $\begin{array}{l}\text { Author(s) } \\
\text { and year }\end{array}$ & Crew premium & $\begin{array}{l}\text { Insurance } \\
\text { premium }\end{array}$ & Transit fees & $\begin{array}{l}\text { Fuel consumption } \\
\text { rate }\end{array}$ & Speed & $\begin{array}{l}\text { Capital Cost } \\
\text { Premium } \\
\end{array}$ \\
\hline $\begin{array}{l}\text { Pruyn } \\
(2016)\end{array}$ & N.A. & $\begin{array}{l}\text { P\&I: }+100 \% \\
\text { H\&M: }+200 \% \\
\text { Piracy Premium: } 18 \\
\text { US\$/GT }\end{array}$ & Five scenarios ${ }^{5}$ & $\begin{array}{l}\text { NSR: Ice } 0,1,2:+5 \% \\
\text { Reg-1, Reg-2: same as } \\
\text { SCR }\end{array}$ & $\begin{array}{l}\text { NSR: Ice } 0: 14.3 \mathrm{kts,} \\
\text { Ice } 1,2: 11 \mathrm{kts}, \mathrm{Reg}-1,2 \text { : } \\
9 \mathrm{kts}, \text { SCR: } 14.3 \mathrm{kts}\end{array}$ & $\begin{array}{l}\text { Ice } 0,1,2:+5 \%, \text { Reg-1,2: } \\
\text { same as a non-ice class } \\
\text { vessel }\end{array}$ \\
\hline $\begin{array}{l}\text { Zhao et al. } \\
\text { (2016) }\end{array}$ & $+10 \%$ & $\begin{array}{l}\text { H\&M : }+100 \% \\
\text { P\&I: }+25 \%\end{array}$ & $\begin{array}{l}\text { Three hypothetical } \\
\text { scenarios based on } \\
\text { historical data }\end{array}$ & $\begin{array}{l}\text { SCR: } 0.3 \mathrm{t} / \mathrm{nm} \\
\text { NSR (ice water): } 0.5 \\
\text { t/nm }\end{array}$ & N.A. & $+20 \%$ \\
\hline Zhao and Hu (2016) & $\begin{array}{l}+14 \% \text { premium incl. in } \\
\text { Operating Costs }\end{array}$ & $\begin{array}{l}+14 \% \text { premium incl. in } \\
\text { Operating Costs }\end{array}$ & Official NSRA fees & $\begin{array}{l}\text { Same in both NSR \& } \\
\text { SCR }\end{array}$ & NSR \& SCR: 12.5 kts & N.A. \\
\hline Zhang et al. (2016) & $\begin{array}{l}\text { Containerships: } \\
+10 \% \\
\text { Oil Tankers: } \\
+20 \% \text { premium incl. } \\
\text { in Operating Costs }\end{array}$ & $\begin{array}{l}\text { Containerships: } \\
\text { H\&M: } 50 \% \text {, P\&I: } 25 \% \\
\text { Oil Tankers: } \\
\text { +20\% premium incl. } \\
\text { in Operating Costs }\end{array}$ & $5 \mathrm{US} \$ / \mathrm{GT}$ & $\begin{array}{l}+30 \% \text { for both } \\
\text { Containerships and Oil } \\
\text { Tankers }\end{array}$ & $\begin{array}{l}\text { Containerships: } \\
\text { NSR: } 12 \text { kts on ice } \\
\text { SCR: } 14.4-17.7 \text { kts } \\
\text { Tankers: } \\
\text { NSR: } 9.4 \text { kts on ice } \\
\text { SCR: } 15-15.5 \mathrm{kts}\end{array}$ & $\begin{array}{l}+30 \% \text { for both } \\
\text { Containerships and Oil } \\
\text { Tankers }\end{array}$ \\
\hline $\begin{array}{l}\text { Faury and Cariou } \\
\text { (2016) }\end{array}$ & $\begin{array}{l}+20 \% \text { premium incl. } \\
\text { in Operating Costs }\end{array}$ & $\begin{array}{l}+20 \% \text { premium incl. } \\
\text { in Operating Costs }\end{array}$ & Official NSRA fees ${ }^{6}$ & $+5.1 \%$ & $\begin{array}{l}\text { SCR: } 14.5 \mathrm{kts} \text {; NSR } \\
\text { (Depending on the } \\
\text { month and zone) }\end{array}$ & N.A. \\
\hline Chou et al. (2017) & N.A. & N.A. & N.A. & N.A. & $\begin{array}{l}\text { NSR: } 17.71 \mathrm{kts} \\
\text { SCR: } 20 \mathrm{kts}\end{array}$ & N.A. \\
\hline Schröder et al. (2017) & N.A. & N.A. & N.A. & N.A. & $\begin{array}{l}\text { Oil Tanker: } 16 \mathrm{kts} \text {, } \\
\text { LNG Tanker: } 20 \mathrm{kts} \text { on } \\
\text { open water, } 8 \text { \& } 7 \mathrm{kts} \text { on } \\
\text { ice respectively }\end{array}$ & N.A. \\
\hline
\end{tabular}

\footnotetext{
${ }^{5}$ Ice 0: No fees, Ice 1,2: 4 US\$/t, Reg-1: 5 US\$/t, Reg-2: 19 US\$/t below 40,000 DWT \& 16 US\$/t above 40,000 DWT

${ }^{6}$ Independent navigation during September, October \& November
} 


\begin{tabular}{|c|c|c|c|c|c|c|}
\hline $\begin{array}{l}\text { Author(s) } \\
\text { and year }\end{array}$ & $\begin{array}{l}\text { Maintenance } \\
\text { premium }\end{array}$ & Load Factor & Type of vessel & Ice Class & Transport Costs $^{7}$ & $\begin{array}{l}\text { Impact of Ice on } \\
\text { Fuel Consumption }\end{array}$ \\
\hline $\begin{array}{l}\text { Wergeland } \\
\text { (1991) }\end{array}$ & $+23.6 \%$ & $75 \%$ in all scenarios & $\begin{array}{l}20,000 \text { DWT } \\
\text { Norilsk type } \\
\text { Multi-purpose vessel }\end{array}$ & $\begin{array}{l}\text { ULA (PC4/PC5, } \\
\text { IACS } \\
\text { Classification) }\end{array}$ & $\begin{array}{l}\text { Shipowner's \& Cargo } \\
\text { Owner's perspective }\end{array}$ & - \\
\hline $\begin{array}{l}\text { Kondo and Takamasa } \\
\text { (1999) }\end{array}$ & $\begin{array}{l}+50.3 \% \text { (Periodic } \\
\text { Maintenance) }\end{array}$ & $100 \%$ in all scenarios & $\begin{array}{l}1,400 \text { TEU } \\
\text { Nuclear-powered } \\
\text { Containership }\end{array}$ & $\begin{array}{l}\text { Cassette-type } \\
\text { MRX Nuclear- } \\
\text { powered Ice- } \\
\text { breaking ship }\end{array}$ & $\begin{array}{l}\text { Shipowner's \& } \\
\text { Shipper's perspective }\end{array}$ & N.A. \\
\hline Guy (2006) & $\begin{array}{l}+15-200 \% \text { premium } \\
\text { incl. } \\
\text { in Trip Charter costs }\end{array}$ & N.A. & $\begin{array}{l}\text { Panamax Containership \& } \\
\text { Panamax Bulker }\end{array}$ & N.A. & $\begin{array}{l}\text { Shipowner's \& } \\
\text { Charterer's perspective }\end{array}$ & N.A. \\
\hline $\begin{array}{l}\text { Somanathan et al. } \\
\text { (2007) }\end{array}$ & $\begin{array}{l}+ \text { US\$ 650/day } \\
+150 \% \text { (Periodic } \\
\text { Maintenance) }\end{array}$ & $100 \%$ in all scenarios & 4,500 TEU Containership & $\begin{array}{l}\text { CAC } 3 \text { (PC3, } \\
\text { IACS } \\
\text { Classification) }\end{array}$ & Shipowner's perspective & $\bullet$ \\
\hline $\begin{array}{l}\text { Somanathan et al. } \\
\text { (2009) }\end{array}$ & $\begin{array}{l}+150 \% \text { (Periodic } \\
\text { Maintenance) }\end{array}$ & $100 \%$ in all scenarios & N.A. & Same as above & Shipowner's perspective & $\bullet$ \\
\hline $\begin{array}{l}\text { Verny and Grigentin } \\
(2009)\end{array}$ & $+100 \%$ & $\begin{array}{l}\text { Westbound: } 100 \% \\
\text { Eastbound: } 30 \%\end{array}$ & 4,000 TEU Containership & N.A. & Shipowner's perspective & N.A. \\
\hline $\begin{array}{l}\text { Liu and Kronbak } \\
(2010)\end{array}$ & $+100 \%$ & $60 \%$ in all scenarios & 4,300 TEU Containership & $1 \mathrm{~B}(\mathrm{IB})$ & Shipowner's perspective & $\bullet$ \\
\hline $\begin{array}{l}\text { Khon and Mokhov } \\
\text { (2010) }\end{array}$ & N.A. & N.A. & N.A. & N.A. & Charterer's perspective & N.A. \\
\hline Khon et al. (2010) & N.A. & N.A. & Bulk/Containership & "Light ice-class" & Charterer's perspective & N.A. \\
\hline $\begin{array}{l}\text { Schøyen and Bråthen } \\
\text { (2011) }\end{array}$ & $\begin{array}{l}+20 \% \text { incl. in Operating } \\
\text { Costs }\end{array}$ & $100 \%$ in all scenarios & $\begin{array}{l}\text { 40,000 DWT Handymax \& } \\
50-68,000 \text { DWT bulk carriers }\end{array}$ & GL E3 (IA) & Charterer's perspective & N.A. \\
\hline Xu et al. (2011) & N.A. & N.A. & 10,000 TEU Containership & No ice-class & Shipowner's perspective & N.A. \\
\hline Fan et al. (2012) & N.A. & $100 \% ?$ & $\begin{array}{l}\text { 4,400 -12,000 TEU } \\
\text { Containerships }\end{array}$ & N.A. & Shipper's Perspective & N.A. \\
\hline $\begin{array}{l}\text { Song and Zhang } \\
\text { (2013) }\end{array}$ & $+26 \%$ & $100 \%$ & $\begin{array}{l}\text { 120,000 DWT } \\
\text { Aframax tanker }\end{array}$ & $1 \mathrm{~A}(\mathrm{IA})$ & Shipowner's perspective & $\bullet$ \\
\hline $\begin{array}{l}\text { Lasserre } \\
\text { (2014) }\end{array}$ & $\begin{array}{l}+20 \% \text { (Periodic } \\
\text { Maintenance) }\end{array}$ & Shanghai-Yokohama9 & 4,500 TEU Containership & 1AS (IAS) & Shipowner's perspective & $\bullet$ \\
\hline
\end{tabular}

${ }^{7}$ Transport costs are distinguished between "Shipowner's" and "Charterer's" unless otherwise stated.

${ }^{8}$ The ice class in the parenthesis is the Finnish-Swedish equivalent where applicable. IACS classification is used otherwise.

${ }^{9}$ NSR: Westbound: NSR: 70\%, SCR: 87\%, Eastbound: NSR: 45\%, SCR: 60\%, NWP: Westbound: NWP: 45\%, SCR: 87\%, Eastbound: NWP: 70\%, SCR: 60\% 


\begin{tabular}{|c|c|c|c|c|c|c|}
\hline $\begin{array}{l}\text { Author(s) } \\
\text { and year }\end{array}$ & $\begin{array}{l}\text { Maintenance } \\
\text { premium }\end{array}$ & Load Factor & Type of vessel & Ice Class & Transport Costs & $\begin{array}{l}\text { Impact of Ice on } \\
\text { Fuel Consumption }\end{array}$ \\
\hline $\begin{array}{l}\text { Raza and Schøyen } \\
(2014)\end{array}$ & N.A. & $\begin{array}{l}\text { Eastbound: NSR \& } \\
\text { SCR: } 90 \%\end{array}$ & $\begin{array}{l}\text { 150,000 cm/84,682 DWT } \\
\text { LNG Tanker }\end{array}$ & Lloyd's 1A (IA) & Charterer's Perspective & N.A. \\
\hline Lu et al. (2014) & N.A. & $100 \%$ in all scenarios? & $\begin{array}{l}4,500-15,000 \text { TEU } \\
\text { Containership }\end{array}$ & N.A. & Charterer's Perspective & N.A. \\
\hline $\begin{array}{l}\text { Lasserre } \\
(2015)\end{array}$ & $\begin{array}{l}\text { Summer Season \& } \\
\text { Year-round }\end{array}$ & In all scenarios ${ }^{11}$ & 4,500 TEU Containership & $\begin{array}{l}\text { 1AS (IA) } \\
\& \text { PC4 }\end{array}$ & Shipowner's Perspective & N.A. \\
\hline $\begin{array}{l}\text { Furuichi and Otsuka } \\
\text { (2015) }\end{array}$ & N.A. & $\begin{array}{l}\text { Eastbound and } \\
\text { Westbound: } 70 \%\end{array}$ & $\begin{array}{l}4,000 \text { TEU - 15,000 TEU } \\
\text { Containership }\end{array}$ & N.A. & Shipowner's Perspective & $\bullet$ \\
\hline $\begin{array}{l}\text { Fan et al. } \\
\text { (2015) }\end{array}$ & N.A. & $100 \%$ & $\begin{array}{l}1000-14,000 \\
\text { TEU Containerships }\end{array}$ & N.A. & Shipper's Perspective & N.A. \\
\hline Chou et al. (2015) & N.A. & N.A. & $\begin{array}{l}60,000 \text { DWT } \\
\text { Panamax Bulk Carrier }\end{array}$ & N.A. & Charterer's Perspective & N.A. \\
\hline Chang et al. (2015) & N.A. & N.A. & N.A. & N.A. & Charterer's Perspective & N.A. \\
\hline $\begin{array}{l}\text { Cariou and Faury } \\
(2015)\end{array}$ & $\begin{array}{l}+9.5 \% \text { premium incl. } \\
\text { in Operating Costs }\end{array}$ & $\begin{array}{l}80 \% \text { in both the NSR } \\
\text { and SCR }\end{array}$ & $\begin{array}{l}40,000 \text { DWT } \\
\text { Handymax Bulk Carrier }\end{array}$ & $1 \mathrm{~A}(\mathrm{IAS})$ & Shipowner's Perspective & $\bullet$ \\
\hline Lindstad et al. (2016) & N.A. & $100 \% ?$ & $\begin{array}{l}\text { Capesize and Panamax } \\
\text { Bulk Carriers }\end{array}$ & N.A. & Charterer's Perspective & $\bullet$ \\
\hline $\begin{array}{l}\text { Pruyn } \\
(2016)\end{array}$ & N.A. & $100 \% ?$ & $\begin{array}{l}\text { 11 Bulk Carrier sizes } \\
(17,000-289,000 \text { DWT })\end{array}$ & $\begin{array}{l}\text { Ice } 0,1,2, \text { Reg- } \\
1,2 \\
\text { (Hypothetical) }\end{array}$ & $\begin{array}{l}\text { Shipowner's \& Charterer's } \\
\text { perspective }\end{array}$ & - \\
\hline $\begin{array}{l}\text { Zhao et al. } \\
\text { (2016) }\end{array}$ & $+100 \%$ & $\begin{array}{l}\text { Real data based on } \\
\text { COSCO's Asia- } \\
\text { N. Europe Service }\end{array}$ & 4,800 TEU Containership & IA? & Shipowner's Perspective & $\bullet$ \\
\hline Zhao and Hu (2016) & $\begin{array}{l}+14 \% \text { premium incl. } \\
\text { in Operating Costs }\end{array}$ & N.A. & $\begin{array}{l}\text { 19,461 DWT Multi- } \\
\text { purpose/general cargo vessel }\end{array}$ & 1A (IA) & Shipowner's Perspective & N.A. \\
\hline $\begin{array}{l}\text { Zhang et al. } \\
(\mathbf{2 0 1 6 )}\end{array}$ & $\begin{array}{l}\text { Containerships: } \\
+20 \% \text {, Oil Tankers: } \\
+20 \% \text { premium incl. } \\
\text { in Operating Costs }\end{array}$ & $\begin{array}{l}\text { Different levels of } \\
\text { demand (TEUs) on each } \\
\text { port, } 100 \% \text { for oil } \\
\text { tankers }\end{array}$ & $\begin{array}{l}\text { New Panamax \& Panamax, } \\
\text { Containerships } \\
\text { VLCC \& Aframax Oil } \\
\text { Tankers }\end{array}$ & $1 \mathrm{~A}(\mathrm{IA}) ?$ & Shipowner's Perspective & $\bullet$ \\
\hline $\begin{array}{l}\text { Faury and Cariou } \\
(2016)\end{array}$ & $\begin{array}{l}+20 \% \text { premium incl. } \\
\text { in Operating Costs }\end{array}$ & $100 \%$ & $\begin{array}{l}74,997 \text { DWT } \\
\text { Panamax Oil Tanker }\end{array}$ & $1 \mathrm{~A}(\mathrm{IA})$ & Shipowner's Perspective & - \\
\hline Chou et al. (2017) & N.A. & $100 \%$ & 5,551 TEU Containership & N.A. & N.A. & N.A. \\
\hline Schröder et al. (2017) & N.A. & N.A. & $\begin{array}{l}\text { 102,000 DWT Oil Tanker } \\
\text { 115,500 LNG Tanker }\end{array}$ & $\begin{array}{l}\text { 1A (IA) } \\
\& \text { Arc7 (PC3) }\end{array}$ & N.A. & $\bullet$ \\
\hline
\end{tabular}

${ }^{10}$ Summer Season: NSR \& NWP: $+20 \%$, Year-round: NWP: $+150 \%$, NSR: $+25 \%$, (both Periodic Maintenance)

${ }^{11}$ Westbound: NSR \& NWP: 70\%, SCR: 85\%, Eastbound: NSR \& NWP: 50\%, SCR: 75\% 